\title{
A comprehensive data-driven model of cat primary visual cortex.
}

abbreviated title: A comprehensive data-driven model of cat V1.

Ján Antolík ${ }^{* 1,2,3}$, Cyril Monier ${ }^{1}$, Yves Frégnac ${ }^{1}$, and Andrew P. Davison ${ }^{\dagger} \ddagger 1$

${ }^{1}$ Unité de Neurosciences, Information et Complexité (UNIC), CNRS FRE 3693,

Gif-sur-Yvette, France

${ }^{2}$ INSERM UMRI S 968; Sorbonne Universits, UPMC Univ Paris 06, UMR S 968; CNRS, UMR 7210, Institut de la Vision, F-75012, Paris, France

${ }^{3}$ Faculty of Mathematics and Physics, Charles University, Malostranské nám. 25, 11800 , Prague 1, Czechia

November 8, 2019

Number of pages: 54

Number of figures: 10

Number of tables: 2

Number of words in Abstract: 212

Number of words in Introduction: 645

Number of words in Discussion: 1500

\section{Acknowledgments}

This work was supported by the Centre National de la Recherche Scientifique, Paris-Saclay IDEX (NeuroSaclay); the French National Research Agency (Complex-V1); the European Union's Seventh Framework Program under grant agreements 269921 (BrainScaleS) and 604102 (Human Brain Project) and through the project Improvement of internationalization in the field of research and development

(CZ.02.2.69/0.0/0.0/17_050/0008466) at Charles University.

\footnotetext{
${ }^{*}$ Corresponding author: antolikjan@gmail.com

†Corresponding author: andrew.davison@unic.cnrs-gif.fr

¥The authors declare no competing financial interests.
} 


\begin{abstract}
Knowledge integration based on the relationship between structure and function of the neural substrate is one of the main targets of neuroinformatics and data-driven computational modeling. However, the multiplicity of data sources, the diversity of benchmarks, the mixing of observables of different natures, and the necessity of a long-term, systematic approach make such a task challenging. Here we present a first snapshot of a long-term integrative modeling program designed to address this issue: a comprehensive spiking model of cat primary visual cortex satisfying an unprecedented range of anatomical, statistical and functional constraints under a wide range of visual input statistics. In the presence of physiological levels of tonic stochastic bombardment by spontaneous thalamic activity, the modeled cortical reverberations self-generate a sparse asynchronous ongoing activity that quantitatively matches a range of experimentally measured statistics. When integrating feed-forward drive elicited by a high diversity of visual contexts, the simulated network produces a realistic, quantitatively accurate interplay between visually evoked excitatory and inhibitory conductances; contrast-invariant orientation-tuning width; center surround interactions; and stimulus-dependent changes in the precision of the neural code. This integrative model offers numerous insights into how the studied properties interact, contributing to a better understanding of visual cortical dynamics. It provides a basis for future development towards a comprehensive model of low-level perception.
\end{abstract}

\title{
Significance statement
}

Computational modeling can integrate fragments of understanding generated by experimental neuroscience. However, most previous models considered only a few features of neural computation at a time, leading to either poorly constrained models with many parameters, or lack of expressiveness in over-simplified models. A solution is to commit to detailed models, but constrain them with a broad range of anatomical and functional data. This requires a long-term systematic approach. Here we present a first snapshot of such an integrative program: a large-scale spiking model of $\mathrm{V} 1$, that is constrained by an unprecedented range of anatomical and functional features. Together with the associated modeling infrastructure, this study lays the groundwork for a broad integrative modeling program seeking an in-depth understanding of vision.

Keywords - visual cortex, cortical microcircuit, large-scale models, comprehensive modeling, layered network 


\section{Introduction}

A key challenge in neuroscience is consolidation of the multitude of experimental findings into a coherent characterization of cortical processing. Slow progress in such knowledge integration is evidenced by the surprisingly poor state of understanding even in well-explored cortical areas, such as primary visual cortex (V1) (Olshausen 2013).

For example, while V1 circuitry supports many different computations occurring concurrently, such as edge detection, depth processing, and contextual modulation, these have mostly been studied in isolation. Many computational studies have proposed mechanisms to explain V1 phenomena-one at a time-including layer-specific resting-state differences (Potjans and Diesmann 2014), contrast adaptation (Bonin et al. 2005), or orientation tuning invariance (Finn et al. 2007). An alternative to such a unipotent model approach is to propose a common adaptive mechanism capable of explaining multiple V1 properties (e.g. Olshausen and Field 1996 Antolík and Bednar 2011). However, only few concurrent phenomena have been actually demonstrated in such models (but see Zhu and Rozell 2013), despite the potential these approaches hold for knowledge integration. The most comprehensive explanations of V1 function come from a few large-scale models combining well-established cortical mechanisms (Somers et al. 1998 Rangan et al. 2009), but these studies still account for only a few phenomena at a time (see section 3.5).

Failure to address the spectrum of phenomena concurrently leads to under-constrained models, and proposition of numerous alternative explanations for individual phenomena, while informing us little about how different V1 computations are multiplexed such that the same synapses participate in multiple simultaneous calculations. While further reductionist, hypothesis-led research is indisputably required, many of these questions require a systematic, integrative approach to progressively build a unified multi-scale theory of brain function (Sejnowski et al. 1988 Einevoll et al. 2019). A danger in detailed modeling is growth of free parameters, risking over-fitting and consequent lack of explanatory power. However, building and validating a model against a large number of diverse experimental studies adds strong constraints on parameters. Similarly, a multi-scale approach greatly increases the categories of experiments suitable as a source of model constraints (Markram et al. 2015).

In our view, the solution to these challenges is a sustained, collaborative effort to incorporate the full breadth of experimentally established constraints into a single pluripotent model of $\mathrm{V} 1$. While necessarily a long-term project, here we offer a basis for such an integrationist effort: a detailed, biologically realistic model of cat $\mathrm{V} 1$, validated against an unprecedented range of experimental measures, including intra- and extra-cellular functional recordings realized over the years in our laboratory. To support collaborative knowledge integration, the model and all experimental protocols are implemented in an easily extensible simulation framework (Antolík and Davison 2013), and all code and data are published online (http://v1model.arkheia.org).

The model comprises cortical layers 4 and $2 / 3$, within a $4.0 \times 4.0 \mathrm{~mm}$ patch around the area centralis. Intracortical connectivity is based on rules extracted from experimental data (Binzegger et al. 2004): thalamocortical connectivity in Layer 4 follows a push-pull organization (Troyer et al.|1998), while lateral connectivity follows parameters derived from Stepanyants et al. (2008) and Buzás et al. (2006). The model has been probed by the most common stimulation paradigms used in early vision: drifting gratings, masked gratings of variable diameter, and naturalistic movies. Under background thalamic bombardment a spontaneous cortical activity within a physiologically plausible 
resting-conductance regime emerges in the model. Under visual stimulation, the same model exhibits diversity of the interplay between evoked excitation and inhibition; stimulus-locked subthreshold variability; contrast-invariant orientation tuning; size tuning; stimulus-dependent firing precision; and a realistic distribution of Simple/Complex receptive fields.

This study advances our ability to capture the function of V1 within a single model, and provides insights into how the studied properties interact, contributing to a better understanding of cortical dynamics. We demonstrate the utility of the proposed function-driven integrative approach and provide a basis for future development towards a comprehensive model of $\mathrm{V} 1$ and beyond that is grounded in an open-software approach.

\section{Materials and Methods}

The model consists of a retino-thalamic pathway feeding input in the form of spikes to a patch of cat primary visual cortex (see Figure 1) centered at 3 degrees of visual field eccentricity. The model's overall architecture was inspired by a previous rate-based model of V1 development (Antolík and Bednar 2011) and a spiking model of a single cortical column (Kremkow et al. 2016).

\subsection{Choice of animal model and cortical state}

The three animal models most utilized in visual neuroscience are cat, Macaque monkey and, recently, mouse. The most extensive body of data on primary visual cortex has been collected for more than half a century in cat. Several of the most important datasets for this study, such as extensive parametric studies of visual receptive fields, the extents of inter-areal connectivity in V1 (Stepanyants et al. 2008) and the relative strength of the different intra-areal pathways (Binzegger et al. 2004), have been collected in this species. Finally there is greater availability of in-vivo intracellular data, which are critical for constraining our model, from the anesthetized cat (Monier et al. 2008: Fournier et al. 2014. Frégnac and Bathellier 2015, Gerard-Mercier et al. 2016). Furthermore, cat area 17 can be equated with macaque area $\mathrm{V} 1$, based on criteria of relative position in the cortical mantle, internal organization of visual field representations, and trans- and sub-cortical connections. In light of these facts, we have chosen to focus our model on cat visual system, but supplement the model with data from macaque whenever equivalent data from the cat is not available, as the closest available approximation.

Due to advances in optogenetic tools, rodents are becoming the dominant animal model for vision, although this sensory modality does not seem as developed as in felines or primates. Targeting of mouse V1 has both advantages and disadvantages. On the one hand, the rodent does not seem to be a natural choice of model for vision when it is obviously more adapted (in the ecological sense) for the haptic modality or the study of multi-modal integration (Frégnac and Bathellier 2015). Mouse V1 still remains less well characterized, especially functionally, than cat or macaque, and major differences in both anatomical and functional organization in comparison with 'higher' mammals have been shown. First at the thalamic level, a much higher percentage of cells in the rodent compared to higher mammals (with the exception of the marmoset) are binocularly activated and already orientation selective. Furthermore, at the 
cortical level, the salt-and-pepper orientation tuning organization in the rodent vs. push-pull layer 4 organization and columnar organization in higher mammals (Frégnac and Bathellier 2015). It is likely that the relative predominance of thalamic vs. intracortical mechanisms in the genesis of such feature selectivities differs between rodents and higher mammals (Frégnac and Bathellier 2015). This interspecies difference could in fact be more fundamental in nature than initially thought. Indeed, the group of Massimo Scanziani has recently shown in the rodent that one of the higher-order visual areas, which is involved in the perception of moving objects, does not depend on information from V1 at all (Beltramo and Scanziani 2019). Instead, this region, known as the post-rhinal cortex (POR), appears to obtain visual data directly from an evolutionarily ancient sensory processing mediated by pulvinar and superior colliculus.

On the other hand, it remains undisputed that advances in optogenetics are rapidly expanding our knowledge of mouse V1 and the semi-industrialization of data acquisition in this species generates datasets that are particularly suitable for the comprehensive data-driven approaches that we champion. Ultimately, we believe having parallel investigations in different strategic animal models (mouse, cat, macaque) and at different levels of detail is desirable, and, rather than using a single species model where optogenetic tools best apply, a generalization of comparative studies to species where valuable functional descriptions have been obtained will shed more light on the mode of cortical processing across different species (Frégnac 2017).

Another key choice is which dynamical cortical state to model. On the one hand, the in vivo data taken from behaving animals might at first glance be more relevant to a realistic description of brain functions tested in a natural environment. However, this assessment should be revised, since, as multiple studies have shown, cortical responses are highly constrained by the context of the behavior itself and the type of fixation eventually used to stabilize recordings. In particular, visual cortical responses in the rodent visual system are contaminated by the locomotor activity imposed by body stabilization or running (which may be far from a "natural" behavior in the ecological sense (see Wallace et al. 2013), up to the point that pure visually-driven processes can no longer be dissected out from the gain control exerted by running activity. On the other hand, data in the anesthetized state suffer from the additional effects of anesthetics on synaptic transmission. There is however a consensus built on 50 years of sensory electrophysiology to consider that stabilization of brain state by Nembutal, Pentobarbital or Althesin induces a reproducible cortical state as long as the anesthesia level is not too deep, which may otherwise lead to suppression of evoked activity and polysynaptic inhibition, or the induction of slow frequency oscillations. This type of preparation still constitutes a recognized standard for the classical studies of unimodal sensory (visual) processing and RF organizational properties (Simple, Complex, orientation preference map, contrast invariance, length summation) which are the focus of interest of the present study. For these reasons we will constrain the model based on data that come from such not-too-deep anesthetized preparations whenever possible.

\subsection{Software and implementation}

The model is implemented, and all experiments and analysis defined in the Mozaik framework (Antolík and Davison 2013), which is freely accessible at https://github.com/antolikjan/mozaik. The NEST simulator (Gewaltig and Diesmann 2007 version 2.2.1) was used as the simulation engine for all simulations described in this paper. Implicit in the 
integrative program proposed here is that progress requires building on previous steps. For such a long-term coordinated effort across the field to be successful, an appropriate infrastructure of tools will be required to facilitate both the incremental construction and sharing of ever more complex models, and efficient, in-depth, systematic comparison of alternative explanations. To catalyze this process, we have built our model in a recent neural network modeling environment, Mozaik (Antolík and Davison 2013), optimized for efficient specification and reuse of model components, experimental and stimulation protocols and model analysis. We are making the resulting model code available under a liberal open-source license so that anyone can build upon it. Sharing the model code that is defined in the highly modular Mozaik environment makes it particularly straightforward for other researchers interested in analyzing the given model or building upon the work presented here, thus supporting the long-term collaboration necessary for the integrative program. At the same time, although neuroscience should ultimately converge upon a single model, there are many paths through the space of partial models to arrive there, and for the health of the field it is good to have several "competing" models, each of which will make different approximations (Einevoll et al. 2019); nevertheless, all models should pass the same validation tests. We therefore also make available our library of integration tests via a dedicated data store (http://v1model.arkheia.org) implemented in the recent Arkheia framework (Antolík and Davison 2018), developed to facilitate sharing model and virtual experiment specifications. The integration tests are structured so as to be independent of the model specification, and their ready-to-use implementation in the Mozaik environment is provided.

\subsection{Parameters choice and model validation}

The level of biological detail of the presented model implies a substantial set of model parameters that have to be determined. The primary challenge is to constrain all model parameters sufficiently to prevent model over-fitting and consequent misinterpretation of the simulation results. The process of parameter determination was divided into two stages: (i) parameter value/range determination based on anatomical or physiological measurements existing in the literature, (ii) parameter tuning in the context of the set of functional features the model seeks to reproduce.

In stage one, having selected the lightly-anesthetized cat preparation as the model target, we have first compiled a list of experimental studies relevant to the design of our model that used the target preparation, and determined the parameter values or ranges for all model parameters for which data existed in this body of literature. Through this process we have obtained constraints for an absolute majority of model parameters: (i) physiological parameters of the neural model were primarily based on intracellular patch clamp recordings by Monier et al. (2008) (see section 2.4.1). (ii) spatial distributions of local connectivity between different cortical layers and neural types were determined by a careful re-analysis of cat anatomical data from Stepanyants et al. (2009) (see section 2.4.4), (iii) functional specificity of layer 2/3 connectivity was determined based on Buzás et al. (2006) (see section 2.4.5), and (iv) synaptic delays were selected based on a set of paired recording studies (see section 2.4.7).

In the ideal case, stage one would be sufficient to fully determine the model parameterization, but as is universally the case for biologically detailed models of neural systems, the current experimental evidence is insufficient for precise determination of every single model parameter. For example, in this study we hypothesize the existence of push-pull 
bioRxiv preprint doi: https://doi.org/10.1101/416156; this version posted November 8, 2019. The copyright holder for this preprint (which was not certified by peer review) is the author/funder, who has granted bioRxiv a license to display the preprint in perpetuity. It is made available under aCC-BY 4.0 International license.

connectivity among Layer 4 neurons, as it is the only plausible explanation for the anti-phase relationship between excitatory and inhibitory conductances in Simple cells (Martinez et al.2005. Baudot et al.2013) proposed to date (see section 2.4.5. As this circuitry has not been directly probed, its hypothetical parameters are unknown. Another poorly constrained parameter is the unitary synaptic strength between neurons. Existing experiments have reported a wide range of values, only indicative of the order-of-magnitude of the synaptic strength, and a systematic identification of the synaptic strength with respect to pre- and post-synaptic neuron type and layer membership is completely missing (see section 2.4.6). Furthermore, it is important to emphasize that for many parameters the multiple studies that have measured them report considerably different values, and thus stage one only provided us with a plausible range rather than an exact value.

Therefore, in stage two, we have proceeded to fine tune the parameters that were not fully determined by stage one of the process. Due to the computational complexity of the model and the number of remaining open parameters this was a manual interactive process. The tuning was guided primarily by the goal of achieving stable dynamics and physiologically plausible levels of intra and extra cellular signals (conductances, membrane potential and spikes) in the spontaneous condition. In our experience, achieving physiological behavior in the spontaneous state secured physiological behavior also in the evoked conditions. While the model dynamics were robust to many of the parameters, we noticed that the model was particularly sensitive to parameters that governed the balance between excitation and inhibition, such as the different synaptic strengths or those influencing neural excitability. The following sections contain a detailed justification of all key model parameters, and a full machine-readable parameterization, that in conjunction with the associated software secures effortless reinstantiation of the entire model, is available in the dedicated web-store (see section 2.2).

Having instantiated the model based on the parameterization determined in the process described above, the next step is to validate the model against as broad a set of experimental findings as possible. Acknowledging the finite manpower resources, and the fact that the integrative program is inherently an incremental process, we have identified a priority list of V1 phenomena to be addressed in this first snapshot. These validation tests were strategically selected to cover most of the most common stimulation paradigms (the stimuli represent a superset of visual stimulation protocols used in our own lab (Baudot et al. 2013) and in the Allen Institute's Brain Observatory (Allen Brain Observatory contributors 2016)) and a broad range of the most salient and well established functional features of early visual system, and thus form a comprehensive battery of validation tests for systematic assessment of early visual system models. At this point it is important to emphasize that the present model does not represent the primary visual cortex of a single cat or even data from a single experiment, rather it represents a generic model of cat V1 that integrates knowledge obtained in a vast number of different experiments themselves spread over an even larger number of individual cat brains. Crucially, many of the targeted features were explored and quantified in several past experimental studies, which have, however, often reported substantially different measurement outcomes. This variability reflects the inherent biological inter-subject brain variability as well as minor differences between the experimental conditions that are often impossible to identify. As a consequence, it is not possible (nor reasonable) to build a single fitness function that would express 'goodness' of the model in one single number, and that could be numerically optimized. Rather, for each of the different model tests, the experimental data outline the range of values 
within which it is reasonable to expect the given measurement outcome to lie. Such a looser nature of the available constraints is, however, compensated by their great number and heterogeneity (different stimuli, different spatial and temporal scales), which ensures substantial constraining pressure on the model. In the results we thus systematically review all the selected measures, identify the ranges dictated by existing experimental evidence and evaluate how the equivalent measurement outcomes in the model conform to them.

It is important to emphasize that this work fully subscribes to the integrative data-driven approach in that it maximally utilized the existing data in the targeted system, and only resorted to alternative options if data were not available. The broad range of stimulation protocols and functional features that the models is tested against ensures that this fitting process avoids major over-fitting that could skew the interpretation of the results.

\subsection{V1 model}

The cortical model corresponds to layers 4 and $2 / 3$ of a $4.0 \times 4.0 \mathrm{~mm}$ patch of cat primary visual cortex, and thus given the magnification factor of 1 at 3 degrees of visual field eccentricity (Tusa et al. 1978), covers roughly $4.0 \times 4.0$ degrees of visual field. It contains 65000 neurons and $\sim 60$ million synapses. This represents a significant down-sampling $(\sim 10 \%)$ of the actual density of neurons present in the corresponding portion of cat cortex (Beaulieu and Colonnier 1989) and was chosen to make the simulations computationally feasible. The neurons are distributed in equal quantities between the simulated Layer 4 and Layer 2/3, which is consistent with anatomical findings by Beaulieu and Colonnier (1989) showing that in cat primary visual cortex approximately the same number of neurons have cell bodies in these two cortical layers. Each simulated cortical layer contains one population of excitatory neurons (corresponding to spiny stellate neurons in Layer 4 and pyramidal neurons in Layer 2/3) and one population of inhibitory neurons (representing all subtypes of inhibitory interneurons) in the ratio 4:1 (Beaulieu et al. 1992 Markram et al. 2004).

We model both the feed-forward and recurrent V1 pathways; however, overall the model architecture is dominated by the intra-cortical connectivity, while thalamocortical synapses constitute less than $10 \%$ of the synaptic input to Layer 4 cells (see Section 2.4.3), in line with experimental evidence (Da Costa and Martin 2011). The thalamic input reaches both excitatory and inhibitory neurons in Layer 4 (see Figure 1 1 FF). In both cortical layers we implement short-range lateral connectivity between both excitatory and inhibitory neurons, and additionally in Layer 2/3 we also model long range excitatory connections onto other excitatory and inhibitory neurons (Stepanyants et al. 2008 Buzás et al. 2006. Angelucci et al. 2002) (see Figure 1 $1 \mathrm{AB}$ ). Layer 4 excitatory neurons send narrow projections to Layer $2 / 3$ neurons (see Figure 1 1 ). In this version, the model omits the infra-granular Layers 5 and 6 as well as the cortical feedback to perigeniculate nucleus (PGN) and lateral geniculate nucleus (LGN), but these issues are being investigated in a follow-up study (preliminary results in Guarino et al. 2017).

\subsubsection{Neuron model}

All neurons are modeled as single-compartment exponential integrate-and-fire neurons (ExplF; Eq. 1). This choice had several motivations. The ExpIF scheme is computationally efficient and offers more realistic membrane potential time courses than simpler integrate-and-fire schemes (Fourcaud-Trocmé et al. 2003). We will show here how the dynamics 


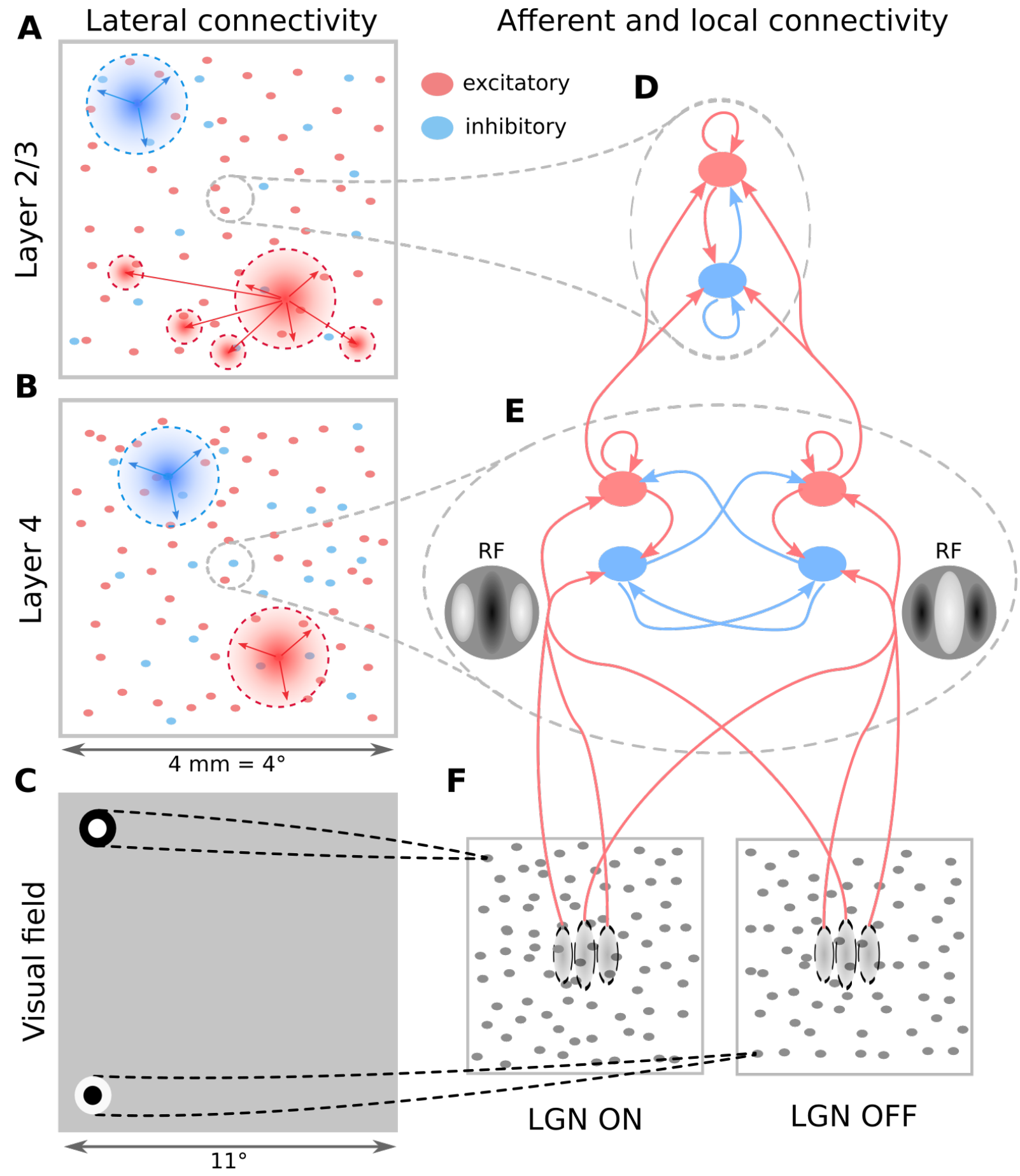

Figure 1: The model architecture. (A-B) Layer $2 / 3$ and Layer 4 lateral connectivity. All cortical neuron types make local connections within their layer. Layer $2 / 3$ excitatory neurons also make long-range functionally specific connections. For the sake of clarity $A, B$ do not show the functional specificity of local connections and connection ranges are not to scale. (C) Extent of modeled visual field and example of receptive fields (RFs) of one ON and one OFF-center LGN relay neuron. As indicated, the model is retinotopically organized. The extent of the modeled visual field is larger than the corresponding visuotopic area of modeled cortex in order to prevent clipping of LGN RFs. (D) Local connectivity scheme in Layer 2/3: connections are orientationbut not phase-specific, leading to predominantly Complex cell type RFs. Both neuron types receive narrow connections from Layer 4 excitatory neurons. (E) Local connectivity in Layer 4 follows a push-pull organization. (F) Afferent RFs of Layer 4 neurons are formed by sampling synapses from a probability distribution defined by a Gabor function overlaid on the ON and OFF LGN sheets, where positive parts of the Gabor function are overlaid on ON and negative on OFF-center sheets. The ON regions of RFs are shown in white, OFF regions in black. 
of sub-threshold signals, including the excitatory and inhibitory synaptic conductances, provide an important means for constraining the model, which thus excludes usage of current-based synapse models. The particular choice of the exponential variant of the conductance-based integrate-and-fire scheme was motivated by our observations that the variable effective threshold in this neural model secures more stable asynchronous behavior than the simpler fixedthreshold variants. Finally, we chose not to pursue more detailed representations of single neuron dynamics, such as a full morphological representation or channel kinetics, for two main reasons: (i) the neuron counts necessitated by the cortical area considered, and the length of the stimulation paradigms targeted in this study would make simulations with such detailed neural models nearly intractable on currently available computing resources, (ii) the understanding of the role of such low-level neural features in cortical computation is still in its relative infancy, especially in terms of direct consequences on the most salient computations performed in V1 that we have targeted here, thus rendering the need for knowledge integration at this level less important, and (iii) lack of sufficient detailed reconstructions of neurons in cat V1 with associated intracellular functional data (Ascoli et al. 2007) and lack of detailed characterization of ion channel kinetics and ion channel dendritic distributions in cat.

The time course of the membrane potential $V(t)$ of all modeled neurons is governed by:

$$
\tau_{\mathrm{m}} \frac{\mathrm{d} V}{\mathrm{~d} t}=-\left(V-E_{\mathrm{L}}\right)+\Delta_{T} \exp \left(\frac{V-V_{T}}{\Delta_{T}}\right)+R_{\mathrm{m}} g_{\mathrm{exc}}\left(E_{\mathrm{exc}}-V\right)+R_{\mathrm{m}} g_{\mathrm{inh}}\left(E_{\mathrm{inh}}-V\right)
$$

where $g_{\mathrm{exc}}$ and $g_{\mathrm{inh}}$ are the incoming excitatory and inhibitory synaptic conductances (see Section 2.4.6 for more details). Spikes are registered when the membrane potential crosses the $-40 \mathrm{mV}$ threshold, at which time the membrane potential is set to the reset value $V_{\mathrm{r}}$ of $-55 \mathrm{mV}$. Each spike is followed by a refractory period during which the membrane potential is held at $V_{\mathrm{r}}$. For all simulated neurons $E_{\mathrm{L}}$ was set to $-70 \mathrm{mV}$ and $V_{T}$ to $-53 \mathrm{mV}$. The membrane resistance in cat $\mathrm{V} 1$ in the absence of synaptic activity has been estimated to be on average $\sim 250 \mathrm{M} \Omega$ (Monier et al. 2008). To reflect these findings we set the membrane resistance of all cortical neurons $R_{\mathrm{m}}$ to $250 \mathrm{M} \Omega$. We set the membrane time constant of excitatory neurons to $15 \mathrm{~ms}$, and of inhibitory neurons to $10 \mathrm{~ms}$, close to values observed experimentally in cat V1 (Monier et al. 2008). The refractory period is set to $2 \mathrm{~ms}$ and $0.5 \mathrm{~ms}$ for excitatory and inhibitory neurons respectively. Overall these neural parameter differences between excitatory and inhibitory neurons reflect the experimentally observed greater excitability and higher maximum sustained firing rates of inhibitory neurons (McCormick et al. 1985). The excitatory and inhibitory reversal potentials $E_{\text {exc }}$ and $E_{\text {inh }}$ are set to $0 \mathrm{mV}$ and $-80 \mathrm{mV}$ respectively in accordance with values observed experimentally in cat V1 (Monier et al. 2008). The threshold slope factor $\Delta_{T}$ was set to $0.8 \mathrm{mV}$ for all neurons based on fits to pyramidal neuron data (Naud et al. 2008).

\subsubsection{Thalamo-cortical model pathway}

All neurons in the model Layer 4 receive connections from the model LGN (see Section 2.5). For each neuron, the spatial pattern of thalamo-cortical connectivity is determined by a Gabor distribution (Eq. 2), inducing the elementary RF properties in Layer 4 neurons (Troyer et al. 1998 see Figure 1 FF). 


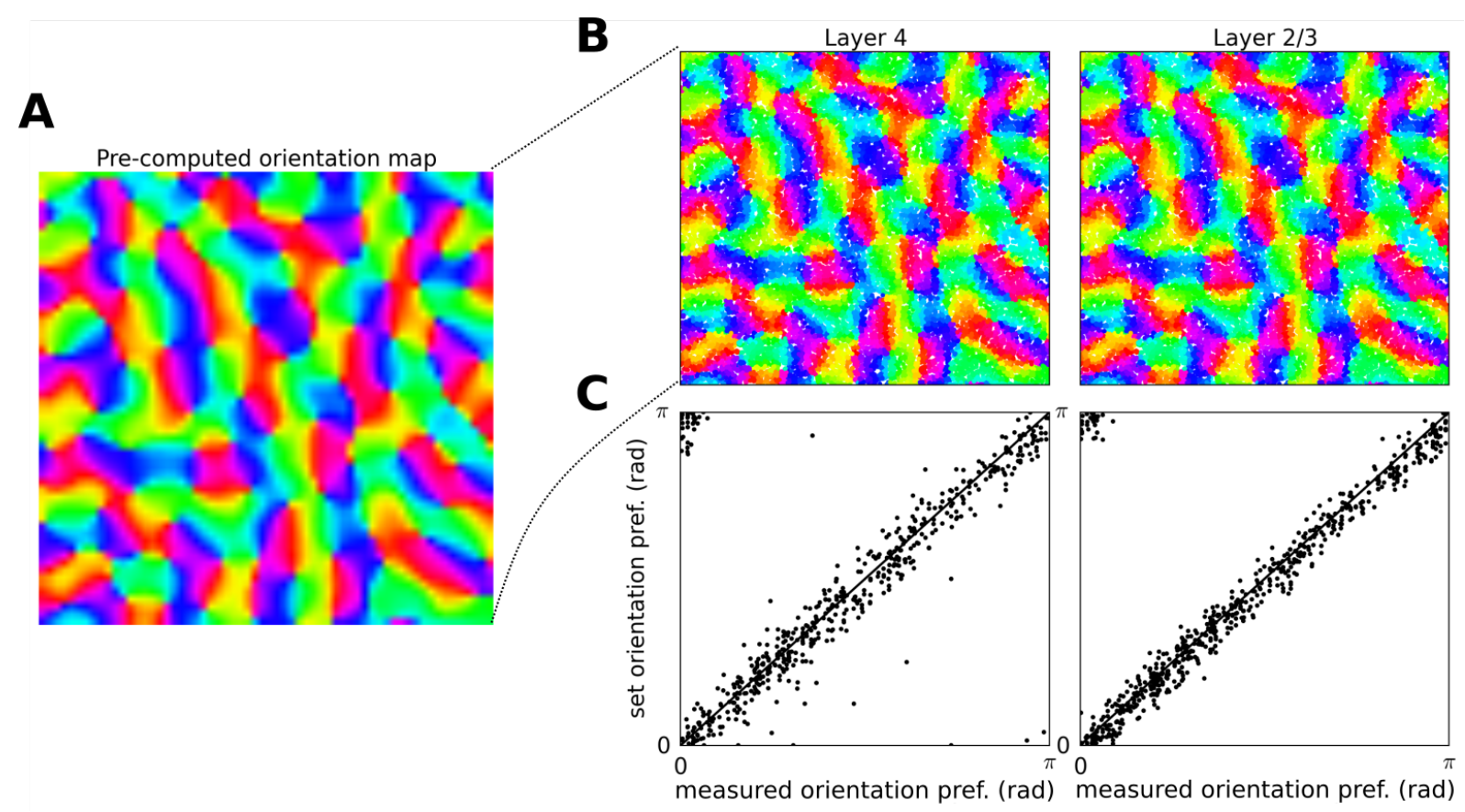

Figure 2: The induction of orientation maps in the model. (A) The precomputed orientation map that serves as a template based on which orientation of the cortico-thalamic connectivity of Layer 4 neurons is determined. (B) The orientation assigned to neurons in the two layers based on their position within the overlaid template orientation map. (C) The comparison between the orientation preference assigned to the neurons via the template ( $y$ axis) and the orientation preference measured using the sinusoidal grating protocol ( $x$ axis; see Section 2.7).

$$
\begin{aligned}
g(x, y, \lambda, \theta, \psi, \sigma, \gamma) & =\exp \left(\frac{x^{\prime 2}+y^{\prime 2} \gamma^{2}}{2 \sigma^{2}}\right) \cos \left(2 \pi x^{\prime} \lambda+\psi\right) \\
x^{\prime} & =x \cos \theta+y \sin \theta \\
y^{\prime} & =-x \sin \theta+y \cos \theta
\end{aligned}
$$

For individual neurons the orientation $\theta$, phase $\psi$, size $\sigma$, frequency $\lambda$ and aspect ratio $\gamma$ of the Gabor distribution are selected as follows. To induce functional organization in the model, we use an existing model of stimulus dependent orientation map development (Antolík and Bednar 2011) that uses Hebbian learning to compute a stabilized link map that conditions an orientation (see Figure 24). Such a pre-computed orientation map, corresponding to the $4.0 \times 4.0 \mathrm{~mm}$ of simulated cortical area, is overlaid onto the modeled cortical surface, thereby assigning each neuron an orientation preference $\theta$ (see Figure $2 \mathrm{~B}$ ). The phase $\psi$ of the Gabor distribution is assigned randomly, in line with the experimental evidence suggesting no clustering of spatial phase in cat V1 (Ziskind 2013). For the sake of simplicity, the remaining parameters are set to constant values, matching the average of measurements in cat V1 RFs located in the para-foveal area. Taking into consideration the estimated mean RF diameter at para-foveal eccentricities (Wilson and Sherman 1976), and assuming that the measured RF perimeter corresponds to the $3 \sigma$ envelope of the Gabor function, we set the size $\sigma$ parameter to 0.17 degrees of visual field. The spatial frequency $\lambda$ was set to $0.8 \mathrm{~Hz}$ based on measurements by Movshon et al. (1978). Finally, studies have produced wide range of estimates for the mean of the 
bioRxiv preprint doi: https://doi.org/101101/416156; this version posted November 8, 2019. The copyright holder for this preprint (which was not certified by peer review) is the author/funder, who has granted bioRxiv a license to display the preprint in perpetuity. It is made available under aCC-BY 4.0 International license.

RF aspect ratio, ranging from 1.7 to 5 (Payne and Peters 2001). We have chosen to set the value of the $\gamma$ parameter to 2.5 , which is at the lower end of this range, as studies restricted to only layer 4 cells produced such lower estimates.

\subsubsection{Cortico-cortical connectivity}

The number of synaptic inputs per single neuron in primary visual cortex of cat has been estimated to be approximately 5800 (Beaulieu and Colonnier 1985). While it is clear that a substantial portion of these synapses come from outside V1, the exact number has not yet been established. A recent investigation by Stepanyants et al. (2009) found that even for a cortical section $800 \mu \mathrm{m}$ in diameter $76 \%$ of synapses come from outside of this region, while a rapid falloff of bouton density with radial distance from the soma has been demonstrated in cat V1 (Buzás et al.|2006). Furthermore, V1 receives direct input from a number of higher cortical areas including V2, V3, V4, V5/MT, MST, FEF, LIP and inferotemporal cortex, as well as other sub-cortical structures. It has been estimated that feedback from V2 alone accounts for as many as $6 \%$ and $8 \%$ of synapses in supra-granular layers of macaque and rat V1 respectively (Budd 1998 Johnson and Burkhalter 1996). Altogether it is reasonable to extrapolate from these numbers that as many as $50 \%$ of the synapses in layers 4 and $2 / 3$ originate outside of the area.

The next important consideration when designing the basic connectivity parameters was the reliability of synaptic transmission in cortex. Cortical synapses have been shown to fail to transmit arriving action potentials, and even though this is a complex context-dependent phenomenon, past studies have shown that in cortex typically every other pre-synaptic action potential fails to evoke a post-synaptic potential (Stratford et al. 1996 Allen and Stevens 1994). This is an important phenomenon as it implies a major change to the overall magnitude of synaptic input that can be expected to arrive into individual cells. It would, however, be computationally expensive to explicitly model the synaptic failures, and so to account for the loss of synaptic drive due to the synaptic transmission failures we have factored it in to the number of simulated synapses per neuron. Thus, taking into account the average number of synaptic inputs (5800), and the estimates of the proportion of extra-areal input (50\%) and of the failure rates of synaptic transmission (50\%) we decided to model 1000 synaptic inputs per modeled excitatory cell. Inhibitory neurons receive $20 \%$ fewer synapses than excitatory neurons to account for their smaller size, but otherwise synapses are formed proportionally to the two cell type densities. $20 \%$ of synapses from Layer 4 cells are formed on the Layer $2 / 3$ neurons. In addition Layer 4 cells receive 110 additional thalamo-cortical synapses (Da Costa and Martin 2011). The synapses are drawn probabilistically with replacement (with functional and geometrical biases described below), and the exact number of synapses according to the numbers above is always established for each neuron, however because we allow formation of multiple synapses between neurons, the exact number of connected neurons and the effective strength of these connections is variable.

The geometry of the cortico-cortical connectivity is determined based on two main principles: the connection probability falls off with increasing cortical distance between neurons (Budd and Kisvárday 2001 Stepanyants et al. 2008. Buzás et al. 2006) (see Figure 1 AB), and connections have a functionally specific bias; specifically pyramidal cells preferentially connect neurons with similar functional properties (Buzás et al. 2006 Ko et al. 2011). The two principles are each expressed as a connection-probability density function, then multiplied and re-normalized to obtain 
the final connection probability profiles, from which the actual cortico-cortical synapses are drawn. The following two sections describe how the two probability density profiles of connectivity are obtained.

Finally, apart from the connectivity directly derived from experimental data, we also modeled a direct feedback pathway from Layer 2/3 to Layer 4. Such direct connections from Layer 4 to Layer 2/3 are rare (Binzegger et al. 2004), however a strong feedback from Layer $2 / 3$ reaching Layer 4 via layers 5 and 6 exists (Binzegger et al. 2004). Since we found that closing of this strong cortico-cortical loop is important for correct expression of functional properties across the investigated layers, and because we decided not to explicitly model the sub-granular layers (see Section 2.4.) we decided to instead model a direct Layer $2 / 3$ to Layer 4 pathway. Since the parameters of this pathway cannot be directly estimated from experimental data, for the sake of simplicity, we have assumed it has the same geometry as the feed-forward Layer 4 to Layer 2/3 pathway (see following two sections).

\subsubsection{Spatial extent of local intra-cortical connectivity}

The exact parameters of the spatial extent of the model local connectivity, with the exception of excitatory lateral connections in Layer 2/3, were established based on a re-analysis of data from cat published in Stepanyants et al. (2008). Let $M_{x y z}^{i j}$ be the probability of potential connectivity (Figure 7 in Stepanyants et al. 2008), of a pre-synaptic neuron of type $i \in\{\mathrm{exc}, \mathrm{inh}\}$ at cortical depth $x$ to other post-synaptic neurons of type $j$ at cortical depth $y$ and lateral (radial) displacement $z$. We reduced the 3 dimensional matrix $M_{x y z}^{i j}$ to a single spatial profile for each pair of layers and neural types (excitatory and inhibitory). This was done as follows. For each possible projection $L_{\text {pre }} T_{\text {pre }} \rightarrow L_{\text {post }} T_{\text {post }}$ where $L$ corresponds to layers $L \in\{4,2 / 3\}$ and $T$ corresponds to neuron type $T \in\{$ exc, inh $\}$ we did the following:

1. Select section of $M_{x y z}^{T_{\mathrm{pre}} T_{\mathrm{post}}}$ along the depth dimensions $x, y$ corresponding to the pre-synaptic layer $L_{\mathrm{pre}}$ and post-synaptic layer $L_{\text {post }}$.

2. Average the selected section of $M$ along the the dimensions corresponding to pre- and post-synaptic layer depth, resulting in a vector representing the average lateral profile of potential connectivity of a neuron residing in layer $L_{\text {pre }}$ to neurons residing in layer $L_{\text {post }}$.

3. Normalize the resulting distance profile to obtain the probability density function.

To obtain a parametric representation of the distance connectivity profiles we fitted them with several probability distributions, including Gaussian, exponential and hyperbolic. We found that the best fits were obtained using the zero mean hyperbolic distribution:

$$
p d f(x)=\exp \left(-\alpha \sqrt{\theta^{2}+x^{2}}\right)
$$

which was thus chosen as the parametric representation of the connectivity profiles. The resulting values of the parameters of the fitted hyperbolic distributions, for all combinations of pre- and post- synaptic layers and neuron types, which were used to generate the local connectivity distance dependent profiles in the model, can be found in Table 1

Finally, the Stepanyants et al. (2008) study reflects only the local connectivity, due to it depending on neural reconstruction in slices which cut off distal dendrites and axons further than $500 \mu \mathrm{m}$ from the cell body, and also 


\begin{tabular}{l|cccc|cccc} 
& \multicolumn{5}{|c|}{$\alpha$} & \multicolumn{4}{c}{$\theta$} \\
& L4 Exc & L4 Inh & L23 Exc & L23 Inh & L4 Exc & L4 Inh & L23 Exc & L23 Inh \\
\hline L4 Exc & 0.0139 & 0.0148 & 0.0174 & 0.0197 & 207.7 & 191.8 & 154.4 & 131.5 \\
L4 Inh & 0.0126 & 0.0119 & & & 237.5 & 256.4 & & \\
L23 Inh & & & 0.0149 & 0.0150 & & & 189.5 & 188.61
\end{tabular}

Table 1: The parameters of hyperbolic profiles (Eq. 3) of potential connectivity derived from Stepanyants et al. (2008) used to generate the distance dependent profile of local connectivity in the model. The parameters for pathways not present in the model are not included in the table.

ignores any potential functional biases. In cat Layer $2 / 3$, unlike in Layer 4 , excitatory neurons send long-range axons up to several millimetres away, synapsing onto other excitatory and inhibitory cells (Angelucci et al. 2002 Buzás et al. 2006). To account for this long-range connectivity in Layer $2 / 3$ we follow the observation by Buzás et al. (2006), that the density of boutons across the cortical surface originating from the lateral connectivity of a small local population of stained Layer 2/3 neurons can be well approximated by the sum of two Gaussian distributions, one short range and isotropic and one long-range and orientation specific (see Section 2.4.5). Thus we model the lateral distribution of the Layer $2 / 3$ excitatory connections as $G\left(\sigma_{s}\right)+\alpha G\left(\sigma_{l}\right)$, where $G$ is a zero mean normal distribution, $\sigma_{s}=270 \mu \mathrm{m}$ and $\sigma_{l}=1000 \mu \mathrm{m}$ are the short and long-range space constants chosen in-line with Buzás et al. (2006), and $\alpha=4$ is the ratio between the short-range and long-range components. Another advantage of the Buzás et al. (2006) model based approach to quantifying the later likelihood of connectivity is that it also takes into account the functional biases that take place in Layer 2/3. How we incorporate these functional biases into our model is described in the following section.

\subsubsection{Functionally specific connectivity}

Unfortunately, the functional connectivity in V1 is still poorly understood. Experimental studies have shown that local connections in cat are diffuse, without a strong orientation bias (Buzás et al. 2006), while long-range connections have a moderate bias towards iso-orientation configurations (Buzás et al. 2006. Gerard-Mercier et al. 2016). Recent more detailed studies of local connectivity in mice have shown that local connections also have a weak bias towards connecting neurons with similar receptive field properties (Ko et al. 2011 Lee et al. 2016). Furthermore, the antiphase relationship between excitatory and inhibitory synaptic conductances found in a majority of cat V1 Simple cells (Martinez et al. 2005: Baudot et al. 2013) can be interpreted as an indirect evidence for inhibitory inputs to be biased towards neurons with anti-correlated RFs (i.e. co-tuned but anti-phase; although inhibitory conductance shows greater diversity of orientation tuning relative to spike-based preference (Monier et al. 20032008 Fournier et al. 2014)). Overall, these anatomical and functional findings point to a weak tendency of excitatory neurons towards connecting nearby neurons of similar receptive field properties while this bias increases somewhat for more distant post-synaptic neurons, and within Layer 4 Simple cells for inhibitory neurons to exhibit weak bias towards connecting to neurons with anti-correlated RFs.

Within Layer 4, the above findings point to the push-pull scheme of connectivity that has been demonstrated to explain a range of phenomena related to orientation tuning (Troyer et al. 1998) (see Figure 1 1 ). To implement this connectivity in our model we have performed the following process. For each pair of Layer 4 neurons the correlation $c$ 
between their afferent RFs (i.e. RF defined only by the afferent inputs from thalamus) is calculated. The connectivity likelihood for a given pair of neurons is then given by $\frac{1}{\sigma \sqrt{2 \pi}} e^{-(c-\mu)^{2} / 2 \sigma^{2}}$ where $\sigma=1.4$ and $\mu=1$ if the pre-synaptic neuron is excitatory or $\sigma=1.4$ and $\mu=-1$ if inhibitory.

In cat cortex excitatory neurons send long-range connections spanning up to $6 \mathrm{~mm}$ along the cortical distance to both excitatory and inhibitory neurons, preferentially targeting those with similar orientation preference (Buzás et al. 2006). To reflect this connectivity in the model we define the connectivity likelihood between pairs of neurons in Layer $2 / 3$ as $\frac{1}{\sigma \sqrt{2 \pi}} e^{-(\Delta o)^{2} / 2 \sigma^{2}}$ where the $\Delta o$ is the difference between the orientation preference of the two neurons, and $\sigma$ is set to 1.4. The connectivity likelihoods described above are renormalized for each neuron to obtain probability density functions. Note that only the long-range component of the Layer $2 / 3$ model is multiplied by this functionally specific bias, while the short-range component remains non-specific (see Section 2.4.4). Overall this parameterization leads to only weak bias towards co-tuned connections in both simulated cortical layers.

We have chosen to model only a weak bias of cortical neurons towards connecting to co-tuned neighbors, which is supported by current data. It should however be emphasized that the effective functional bias is higher: the presence of functional maps along the cortical surface implies that even connections from a given neuron that would be drawn based on a functionally nonspecific rule would more likely synapse onto co-oriented neurons. Furthermore it is likely that the functional specificity of cortical connections is higher than the level indicated by the limited number of experimental studies that have been conducted, but some of the specificity has not yet been discovered. Such functional wiring specificities can have genetic but also epigenetic origins, whereby specific salient features of the sensory-motor experience of each individual are imprinted via Hebbian-like learning processes onto the cortical wiring. Therefore, the parameters of model connectivity that govern its stochasticity should be viewed as a combination of the true level of randomness in cortical connectivity with an unbiased representation of the yet to be identified specificities.

The push-pull scheme in Layer 4 is motivated by the fact that it has been shown in multiple studies that in Simple cells in Layer 4, the excitatory and inhibitory conductances are in anti-phase (Martinez et al. 2005, Monier et al. 2008. Baudot et al. 2013), and such correlation based connectivity is the only plausible explanation for this phenomenon to date. On the other hand, the phase non-specificity in Layer $2 / 3$ is the consequence of the lack of phase-selectivity of Complex cells that dominate in this layer. This connectivity scheme is thus consistent with all the current experimental findings, and can be viewed as a self-consistent end-product of Hebbian-like plasticity (anti-Hebbian in the case of inhibitory synapses) and stimulus driven development in V1 (Antolík and Bednar 2011, Kayser and Miller 2002).

\subsubsection{Synapses}

The relatively few studies that have examined in detail the strength of individual synapses in cortex have found that unitary synaptic strengths are generally weak $(<2 \mathrm{nS})$, but broad ranges have been reported (Hoffmann et al. 2015. Cruikshank et al. 2007). While the overall specificity of the synaptic strength between pairs of neural types or layers remains unclear, a recent study in cat V1 has shown that synapses from excitatory onto inhibitory cells are considerably stronger than those targeting excitatory neurons (Hoffmann et al. 2015 Cruikshank et al. 2007). Reflecting these insufficient experimental constraints, the synaptic weights were selected to achieve an overall balance 
between excitation and inhibition that supports reasonable levels of both spontaneous and evoked activity, while being compatible with the limited physiological findings. Specifically, we set the intra-cortical excitatory-to-excitatory synapses to $0.375 \mathrm{nS}$ and excitatory-to-inhibitory neurons to $0.675 \mathrm{nS}$, while all inhibitory synapses are set to $1.575 \mathrm{nS}$.

Furthermore, the thalamo-cortical synapses were set to $1.2 \mathrm{nS}$, reflecting the findings that these synapses tend to be larger (Da Costa and Martin 2011) and more reliable (Stratford et al. 1996) than their intra-cortical counterparts.

Synaptic inputs are modeled as transient conductance changes, with exponential decay with time-constant $\tau_{e}=$ $7.0 \mathrm{~ms}$ for excitatory synapses and $\tau_{i}=11.0 \mathrm{~ms}$ for inhibitory synapses.

We have also modeled synaptic depression for thalamo-cortical, and excitatory cortico-cortical synapses (Abbott 1997) using the model of Markram et al. (1998), while we do not model short term plasticity for inhibitory synapses as it is not well studied. For the thalamo-cortical synapses we assume parameters corresponding to weak depression similar to Banitt et al. (2007) and Kremkow et al. (2016) $\left(\mathrm{U}=0.75, \tau_{\mathrm{rec}}=500 \mathrm{~ms}, \tau_{\mathrm{psc}}=7.0 \mathrm{~ms}\right.$ and $\left.\tau_{\mathrm{fac}}=0 \mathrm{~ms}\right)$. For the cortico-cortical excitatory synapses we assume stronger depression $\left(\mathrm{U}=0.75, \tau_{\mathrm{rec}}=30 \mathrm{~ms}, \tau_{\mathrm{psc}}=7.0 \mathrm{~ms}\right.$ and $\left.\tau_{\text {fac }}=0 \mathrm{~ms}\right)$, in line with Markram et al. (1998).

\subsubsection{Delays}

We model two types of delays in the model circuitry. First are the delays due to the distance dependent propagation that are on the order of several tens of ms. These delays are important for lateral integration of information across cortical columns. To reflect this, for all intra-cortical connectivity a distance-dependent delay with propagation constant of $0.3 \mathrm{~ms}^{-1}$ (Bringuier et al. 1999 Frégnac 2012 Chavane et al. 2011) was used, which corresponds to the slow propagation of action potentials along the intra-V1 (lateral) un-myelinated axons. The delays in the feed-forward thalamo-cortical pathway are drawn from a uniform distribution within the range $(1.4,2.4) \mathrm{ms}$. Second, Ohana et al. (2012) have recently shown that delays of synaptic transmission in cat visual cortex are dependent on both pre- and post-synaptic neural type, with the notable feature of slow excitatory to excitatory and fast excitatory to inhibitory transmission. Distance-dependent axonal propagation delay is unlikely to explain these results as these experiments were performed in nearby neurons (Ohana et al. 2012). These pair-specific synaptic integration delays are in the order of only a few ms, but are important for local integration (within the same column) and for the precise timing of spike control by E/I interaction. Thus, as suggested by Ohana et al. (2012), we have included a constant additive factor in all synaptic delays, specifically $1.4 \mathrm{~ms}$ for excitatory to excitatory synapses, $0.5 \mathrm{~ms}$ for excitatory to inhibitory synapses, $1.0 \mathrm{~ms}$ for inhibitory to excitatory synapses and $1.4 \mathrm{~ms}$ for inhibitory to inhibitory synapses, in line with the quantitative observations by Ohana et al. (2012). We observed that the addition of this neuron-type-dependent delay factor improved the stability of the modeled cortical neural networks, reducing synchronous events during spontaneous activity. We hypothesized that this is due to the ability of inhibition to respond faster to any transient increase in activity in the network due to the shorter excitatory to inhibitory delay. 


\subsection{Input model}

The input model described below corresponds to the whole retino-thalamic pathway. The cortical model corresponds to roughly $4.0 \times 4.0^{\circ}$ of visual field (Figure 1 $\mathrm{CF}$ ). To accommodate the full extents of RFs of neurons at the edges of the model, the LGN model corresponds to $5 \times 5^{\circ}$ of visual field. In the same manner, to accommodate the full extent of RFs of thalamic neurons the overall visual field from which the thalamic model receives input corresponds to $11 \times 11^{\circ}$.

We do not explicitly model the retinal circuitry and use the widely-used center-surround model of receptive fields to simulate the responses of the LGN neurons (Figure 1 1 ). The centers of both ON and OFF LGN neurons RFs are uniformly randomly distributed in the visual space, with density 100 neurons per square degree. Each LGN neuron has a spatiotemporal receptive field, with a difference-of-Gaussians spatial profile and a bi-phasic temporal profile defined by a difference-of-gamma-functions (Allen and Freeman 2006). Due to the relatively small region of visual space our model covers, we do not model the systematic changes in RF parameters with foveal eccentricity (nor, for the sake of simplicity, the natural cell-to-cell variance) and thus assume that all ON and OFF LGN neurons have identical parameters. The exact spatial and temporal parameters have been adopted from Allen and Freeman (2006).

To obtain the spiking output of a given LGN neuron, the visual stimulus, sampled into $7 \mathrm{~ms}$ frames, is convolved with its spatiotemporal receptive field. In addition, saturation of the LGN responses with respect to local contrast and luminance is modeled (Papaioannou and White 1972 Bonin et al. 2005). For simplicity, the local luminance $l l$ is calculated as the mean of luminance values and local contrast $l c$ as the standard deviation of the luminances within the RF of a given neuron. The response of the linear receptive field is separated into a DC (luminance) component $r_{l}$ and a contrast component $r_{c}$. The saturation of the two components is modeled with two Naka-Rushton functions $\alpha_{l} \frac{r_{l}}{1.0+\beta_{l} l l}$ and $\alpha_{c} \frac{r_{c}}{1.0+\beta_{c} l c}$ respectively, where $\alpha$ is the gain and $\beta$ is the saturation parameter of the corresponding component. The parameters $\alpha$ and $\beta$ were empirically adjusted to obtain luminance and contrast response curves whose saturation point and level are within the range of those observed experimentally (Papaioannou and White 1972. Bonin et al. 2005).

The resulting luminance and contrast temporal traces are then summed and injected into integrate-and-fire neurons as a current, inducing stimulus dependent spiking responses. In addition to the stimulus-dependent drive, neurons are also injected with white noise current. The magnitude and variance of this noise is such that neurons fire $\sim 10$ spikes/s in the no stimulus condition (Troyer et al. 1998). This artificially elicited spontaneous discharge, calibrated to reproduce the experimentally observed spontaneous rates, corresponds to the combined effects of the dark discharge of the retina and any other potential intrinsic mechansism of spontaneous activity generation in the thalamus.

\subsection{Stimulation protocols}

One of the most important aspects of this study is the broad range of stimuli and associated experimental protocols with which the model was tested. In order to facilitate rigorous testing of future models, we publish the exact specifications of all stimuli and experimental protocol with ready to use implementations on the http://v1model.arkheia.org website and associated repositories. 
Each stimulation protocol consists of a series of visual stimuli which are interleaved with $150 \mathrm{~ms}$ of $50 \mathrm{~cd} / \mathrm{m}^{2} \mathrm{gray}$ blank stimuli. In all experiments, with the exception of the size tuning protocol (see Section 2.6.3), we recorded spikes from 2337 randomly selected neurons, restricted to the central circular patch (radius of $1 \mathrm{~mm}$ ) of the model to avoid contamination by potential edge-effects due to the finite simulated cortical area. Because the recording of intracellular signals at high temporal resolution for such a long stimulus set as presented in this work is extremely memory consuming, we recorded the membrane potential and excitatory and inhibitory conductances from a subset of 201 neurons confined to the central $0.2 \times 0.2 \mathrm{~mm}$ patch of the simulated cortical space, and whose orientation preference (estimated as the expected orientation preference assigned to the neurons during model generation; see Section 2.4.5) was within 0.2 radians of horizontal. The durations of all visual stimuli are aligned with the $7 \mathrm{~ms}$ frame duration at which the retino-thalamic model operates (see Section 2.5).

\subsubsection{Spontaneous condition}

Spontaneous activity was recorded during the presentation of a constant, blank stimulus of zero luminance lasting 40 seconds. In this condition, the visual stimulus does not contribute any input to the phenomenological models of LGN units (see Section 2.5), and thus corresponds to experimental recording in absolute darkness. Note however that under these conditions, LGN integrates the "dark discharge" from the retina and the thalamic drive in the absence of visual input still bombards the cortex at a significant rate (Ghose and Freeman 1992). This tonic thalamic input is simulated by an additive Gaussian noise matching experimental observation.

\subsubsection{Drifting sinusoidal grating protocol}

Sinusoidal gratings, which represent Fourier inputs in space and time, are the most common stimulus employed for characterizing functional properties of visual neurons (Rust and Movshon 2005). They match the RF profile of the V1 neurons (when optimally parametrized) and thus provide a strong simultaneous feed-forward drive to a substantial portion of $\mathrm{V} 1$ neurons, leading to strong neural responses. They are thus ideal for parametric exploration of elementary functional properties of V1 neurons, such as orientation, contrast or frequency tuning.

The spatial and temporal frequency of the RFs of the modeled LGN neurons (see Section 2.4.2) and of the Gabor distribution template from which thalamo-cortical synapses were sampled were identical. An important consequence of this simplification is that it allowed us to efficiently execute protocols requiring drifting sinusoidal gratings. By employing a full-field stimulus with spatial frequency matching that of the Gabor template $(0.8 \mathrm{~Hz})$ and drifting at 2 $\mathrm{Hz}$, we were in parallel stimulating all cortical neurons with a stimulus with optimal spatial frequency. Due to the lack of any direction preference mechanisms in the model, to further lessen the computational burden of the simulations, we varied the orientation of the gratings only between 0 and 180 degrees, in 8 equal steps. Each grating was shown 10 times for $2058 \mathrm{~ms}$. 


\subsubsection{Size tuning protocol}

A small stimulus can elicit a response from a neuron when presented in its RF, but by definition a neuron will not respond when the same stimulus is presented outside of the RF. However, when there is a stimulus in the RF that itself elicits a certain firing rate from the neuron, additional stimuli presented in the surrounding area can increase or decrease that firing rate. Such contextual effects in early vision are believed to underlie higher-level processes such as contour integration and figure-ground segregation.

Size tuning was measured using drifting sinusoidal gratings of optimal spatial and temporal frequency (see orientation tuning protocol for details), confined to an aperture of variable diameter, which were presented for $2058 \mathrm{~ms}$ and repeated 10 times. The orientation of the gratings was horizontal $\left(0^{\circ}\right)$ and the position was in the center of the simulated visual field. The aperture of the grating was gradually increased from $0^{\circ}$ to $3^{\circ}$ in 12 steps, each presentation lasting $2058 \mathrm{~ms}$ and repeated 10 times.

The center of the modeled cortical area was occupied by a horizontal orientation domain (see Figure 2). During the size tuning protocol we recorded from neurons in this central (horizontal) orientation domain and selected neurons whose receptive fields centers were within $0.1^{\circ}$ from the center of the simulated visual field and whose orientation preference (determined by the orientation tuning protocol described above) was within 0.1 radians from horizontal. This setup allowed us to simultaneously determine the size tuning of the population of neurons reported here, with precision comparable to experimental studies, while greatly reducing the computational resources required for the simulation.

\subsubsection{Natural images with simulated eye-movement protocol}

We have replicated the natural image with simulated eye-movement protocol introduced by Baudot et al. (2013). This protocol emulates the global retinal flow experienced during the exploration of the natural environment, by simulating the retinal impact of visuomotor interaction by imposing shifts and drifts of a static natural scene which reproduce the kinematics of a realistic ocular scanpath (Baudot et al. 2013) recorded in the intact behaving animal. We used the same image as in Baudot et al. (2013), scaled to match the size of the simulated visual space, and the same path. Presentation of the resulting movie lasting 2058 ms was repeated 10 times.

\subsection{Data analysis}

Unless specified otherwise, all tuning curves in this study were calculated as the trial averaged mean firing rate during stimulation with the given stimulus and parameter value (e.g. orientation of sinusoidal grating). Spontaneous activity level was not subtracted, to make the tuning curves comparable to experimental studies (Alitto and Usrey 2004 Cardin et al. 2007). All analog signals (membrane potential, excitatory and inhibitory conductances) were recorded at $1 \mathrm{~ms}$ resolution to achieve manageable data quantities, given the large number of neurons recorded and the length of the stimulation protocols.

To characterise the dynamical state of our network during the spontaneous condition at single cell and population level we have used two widely used descriptors (Kumar et al. 2008 Destexhe 2009). We calculate the irregularity of 
an individual neuron's spike train as the coefficient of variation (CV) of its inter-spike-intervals (ISI):

$$
C V_{I S I}^{2}=\frac{\operatorname{Var}[I S I]}{\langle I S I\rangle^{2}}
$$

where Var corresponds to variance, and \langle\rangle is the mean. To ensure accurate estimation of the irregularity we exclude neurons that fired fewer than 10 spikes during the duration of the spontaneous activity recording. For perfectly regular firing $C V_{I S I}=0$, while a Poisson process yields $C V_{I S I}$ equal to one. Following Kumar et al. (2008) we set the threshold for irregular firing to $C V_{I S I}>0.8$. We assess synchrony in our network by calculating the average cross-correlation (CC) of spike counts $S C_{i} S C_{j}$ between disjoint pairs of all recorded neurons $i, j$.

$$
C C\left[S C_{i}, S C_{j}\right]=\frac{\operatorname{Cov}\left(S C_{i}, S C_{j}\right)}{\sqrt{\operatorname{Var}\left(S C_{i}\right) \operatorname{Var}\left(S C_{j}\right)}}
$$

where Var is again variance and $\mathrm{Cov}$ is covariance of the spike count. The spike counts were calculated by counting the number of spikes within $10 \mathrm{~ms}$ bins.

In order to assess orientation tuning, we followed Nowak et al. (2008) and calculated two complementary measures: the half width at half height $(\mathrm{HWHH})$ and relative unselective response amplitude (RURA). To calculate HWHH we fitted the orientation tuning curves with a Gaussian function (Alitto and Usrey 2004):

$$
R(\phi)=\beta+\alpha \exp \left(\frac{\phi-\phi_{\text {pref }}}{2 \sigma^{2}}\right)
$$

where $R$ is the spiking response of the given neuron to a sinusoidal grating with orientation $\phi, \phi_{\text {pref }}$ is the preferred orientation of the given neuron, $\sigma$ is the width of the tuning, $\beta$ is the baseline activity and $\alpha$ a scale factor. Low responding neurons (less then 1 spike/s at optimal orientation) were excluded from the analysis, as reliable curve fitting was not possible with the amount of recorded data. Furthermore, a small minority of neurons $(<5 \%)$, for which a reliable fit of the Gaussian curve was not possible $(M S E>30 \%$ of the tuning curve variance) were also excluded from this analysis. $\mathrm{HWHH}$ was then calculated as $\sqrt{2 \ln 2} \sigma$. RURA was calculated from the fitted Gaussian parameters according to the following equation (Alitto and Usrey 2004):

$$
R U R A=\frac{\beta}{\beta+\alpha}
$$

The modulation ratio (MR) of spike responses was computed as the ratio of the fundamental Fourier component F1 and DC component F0 of the neuron's peri-stimulus time histogram (PSTH) in response to a drifting sinusoidal grating (Skottun et al. 1991). The PSTH was formed with $10 \mathrm{~ms}$ bins, and spontaneous activity was subtracted prior to MR calculation. The modulation ratio of the membrane potential was calculated analogously as the V1/V0 component ratio of the membrane potential after subtraction of its resting level.

Finally, we also examined the most common manifestation of surround modulation, size tuning. To quantify the parameters of size tuning curves, following Wang et al. (2009), we first fitted the data with the Supplementary Difference of Gaussians (3G) model defined as: 


$$
f(r)=K_{\mathrm{c}} G_{\mathrm{c}}(r)-K_{\mathrm{s}} G_{\mathrm{s}}(r)+K_{\mathrm{cS}} G_{\mathrm{cS}}(r)
$$

where

$$
G_{x}(d)=\left(\frac{2}{\sqrt{\pi}} \int_{0}^{d} e^{-\left(y / w_{x}\right)^{2}} d y\right)^{2}
$$

where $d$ is the stimulus diameter, $K_{\mathrm{c}}$ is the strength of the center, $K_{\mathrm{s}}$ is the strength of the suppressive surround, $K_{\mathrm{cs}}$ is the strength of the counter-suppressive surround (accounting for reduction of length suppression in the far periphery) and $w_{x}$ are the space constants of the corresponding terms. We then performed the following analysis on the fitted curves. First we determined the diameter at which the peak summation occurs. The exact definition of this point in different studies varies slightly, the most important difference being that in some studies it has been computed directly from the raw tuning curves (Sengpiel et al. 1997, Cavanaugh et al. 2002 Jones et al. 2001), whereas in others authors have first fitted the size tuning curve with models (of various kinds) and then derived these points from the fitted model parameters (Ichida et al. 2007 Sceniak et al. 1999 Tailby et al. 2007; Wang et al. 2009). Because the studies testing both approaches found very good agreement between the parameters derived in these two ways, we followed Wang et al. (2009) and determined the parameters from the fitted curves. We excluded low responding neurons (response of less then 2 spikes/s at the optimum size) from the analysis as we have observed poor curve fitting to these size tuning curves due to lack of data. We defined the summation peak $\delta_{\max }$ as the diameter where maximum response is achieved before suppression sets in (we will also refer to this diameter as the maximum facilitation diameter $(\mathrm{MFD})$ ), suppression peak $\delta_{\min }$ as the diameter where the minimum response is achieved across all aperture sizes greater than $\delta_{\max }$, and the counter-suppression peak $\delta_{\mathrm{cs}}$ as the diameter where the maximum response is achieved across all aperture sizes greater than $\delta_{\min }$. This in turn allows us to define the peak summation response $R_{\max }$, peak suppression response $R_{\mathrm{min}}$, and peak counter-suppression response $R_{\mathrm{cs}}$ as the neuron's responses at the corresponding aperture sizes. We can then define the suppression index (SI) analogously to experimental studies (Wang et al. 2009) as:

$$
S I=\frac{R_{\max }-R_{\min }}{R_{\max }}
$$

and the counter-suppression index CSI as:

$$
C S I=\frac{R_{\mathrm{cs}}-R_{\min }}{R_{\max }}
$$

The reliability and the precision of the responses were measured by fitting a Gaussian function to the mean crosscorrelation across trials (Eq. 5) of the spiking responses, and of the sub-threshold membrane potential responses (Baudot et al. 2013). Spikes were removed from the membrane potential prior to the analysis, by replacing the membrane potential within a $5 \mathrm{~ms}$ window centred on each spike with the signal interpolated between the levels of membrane potential before and after the window. The reliability was then defined as the CC peak amplitude at time zero, and the temporal precision by the standard deviation of the Gaussian fit. 
Quantitative values in this article are expressed as mean \pm SEM.

\subsection{Modeling choices}

The choice of the level of modeling detail employed here is what we consider a middle ground, where we neither engage low-level details such as neuronal morphology or detailed channel dynamics, nor opt for high-level approximations such as mean field approaches. Instead, the choice of which experimentally identified neural mechanisms to incorporate in the model is guided by the functional phenomena that are being gradually targeted by the model, a principle naturally stemming from the idea of a long-term incremental integrative approach. The choice of this 'function guided' approach has the following advantages: (i) the most salient features of V1 computation are naturally represented at this level of detail; (ii) this level of detail enables a clear, direct link between the underlying neural substrate and V1 function, (iii) the model remains minimal in the sense that it only incorporates elements necessary for induction of the targeted functional features, (iv) as a platform for future research it facilitates integration across scales by allowing straight-forward expansion both towards low-level features (by simply replacing the neural models with more detailed approximations of ones choice), and high-level features (by expanding the model towards further cortical layers, other cortical and sub-cortical areas and measurements of associated functional phenomena).

The model makes five main simplifications: (i) the omission of layers 5 and 6 (Thomson 2010, 2003), (ii) omission of the cortico-thalamic feedback pathway (Wang et al. 2006 Sillito et al. 1994), (iii) omission of the feedback from higher cortical areas (Angelucci and Bullier 2003 Budd 1998), (iv) reduction of neural dynamics to only two types (excitatory and inhibitory) (Xu and Callaway 2009 Nowak et al. 2003), and (v) the reduced cortical neuron density. The infra-granular layers have been omitted because they are much less understood and there is much less data available for constraining their model. Furthermore, they are believed to be largely involved in sub-cortical projections and feedback projection from higher level cortical areas (Markov et al. 2014), which are another two features not considered here (but see Guarino et al. 2017). The cortico-cortical feedback, mediated partly by long-distance supragranular connections, represents a particularly difficult challenge for modeling, as it in principle requires some form of treatment of all the numerous higher-cortical areas that send feedback to $\mathrm{V} 1$, while relying on a relatively sparse set of findings on the anatomy, physiology and computational principles of these feedback structures. Due to recent methodological advances in selective targeting of different neural subtypes the roles which the different inhibitory neural sub-types play in cortical processing are starting to be uncovered (Pfeffer et al. 2013: Harris and Mrsic-Flogel 2013). This mapping out of the neural sub-type characteristics is, however, still in an early stage, and is largely confined to the mouse animal model, without a clear understanding of how these findings will translate to cortical processing in higher mammals. Undoubtedly, continuing systematic exploration of the neural sub-type specificities, especially if successfully translated into higher mammalian models, would provide an invaluable trove of constraints to be incorporated into the integrative modeling paradigm proposed here. 


\section{Results}

The central goal of this study is to show that a single model of cat primary visual cortex, composed only of elements that do not contradict any existing experimental evidence, can reproduce an unprecedented range of previously identified phenomena, under a broad range of stimulation protocols. In order to present such a heterogeneous set of results in a clear manner, we organize the results such that each sub-section reviews the behavior of the model during a single stimulation protocol and compares it with experimental findings. All results presented here are based on data recorded in a single instantiation of the model.

\subsection{Spontaneous activity}

The model does not include any external sources of variability, with the exception of white noise current injection into LGN cells in order to induce their spontaneous firing at about $10 \mathrm{~Hz}$, in line with recordings from visual thalamus (Ghose and Freeman 1992). This noise represents the combined effect of the dark discharge (Cavaggioni 1968) in retina due to photonic noise or intrinsic properties of retinal ganglion cells (Levine 1991, Field and Rieke 2002, Croner et al. 1993 Salari et al. 2016), and the intrinsic mechanisms generating ongoing activity in thalamus (Rogala et al. 2013). Consequently, since the thalamic inputs to Layer 4 neurons form only a small fraction of the modeled cortical synapses (in line with anatomy), the spontaneous activity (and variability in general) in the model is largely due to the internal dynamics of the model. This is an important factor for measurements that examine changes in cortical variability under different conditions such as those explored in Section 3.4

In the spontaneous condition all modeled excitatory neuron populations fire irregularly (Figure 34 ; Figure 43 : $\mathrm{CV}$ of ISI is above 0.8 in all layers) and asynchronously (Figure 3 $\mathrm{A}$; Figure 4C: the mean cross-correlation of the spike counts in excitatory populations is less then 0.01 ), with only occasional synchronized events, consistent with the spontaneous firing patterns of neurons in cortical slices (Cossart et al. 2003: Shu et al. 2003), awake animals (Esposito et al. 2009: Lee et al. 2006) and up-states in in vivo anesthetized preparations (Esposito et al. 2009: Destexhe and Rudolph-Lilith 2012). The model exhibits slightly higher correlations between the spontaneous responses of inhibitory neurons then excitatory ones (Figure 3A; Figure 4C). Interestingly, higher synchrony among inhibitory neurons (as opposed to excitatory ones) has recently been reported in macaque cortex during slow wave sleep (Le Van Quyen et al. 2016). Overall the spontaneous activity corresponds to the asynchronous irregular (Al) state previously observed in balanced randomly connected network models (Van Vreeswijk and Sompolinsky 1996 Brunel 2000 Vogels and Abbott 2005).

The mean firing rates differ considerably between cell types (Figure $3 \mathrm{~A}$ ). We observe higher mean spontaneous rates in inhibitory populations than in excitatory populations, in line with experimental evidence (Bringuier et al. 1997. Swadlow 2003), while the spontaneous firing rates of the majority of excitatory neurons are low $((<2 \mathrm{~Hz})$, as observed by Monier et al. 2003, Bardy et al. 2006 Figure 4A). The firing rates of individual neurons can differ substantially from the population mean, as shown by the population histograms in Figure $4 \mathrm{G}$. In all four cortical populations, the firing rates closely follow log-normal distributions (Figure $4 \mathrm{G}$ ), a phenomenon previously shown in several cortical areas and species (Hromadka et al. 2008. Buzsáki and Mizuseki 2014). 
A

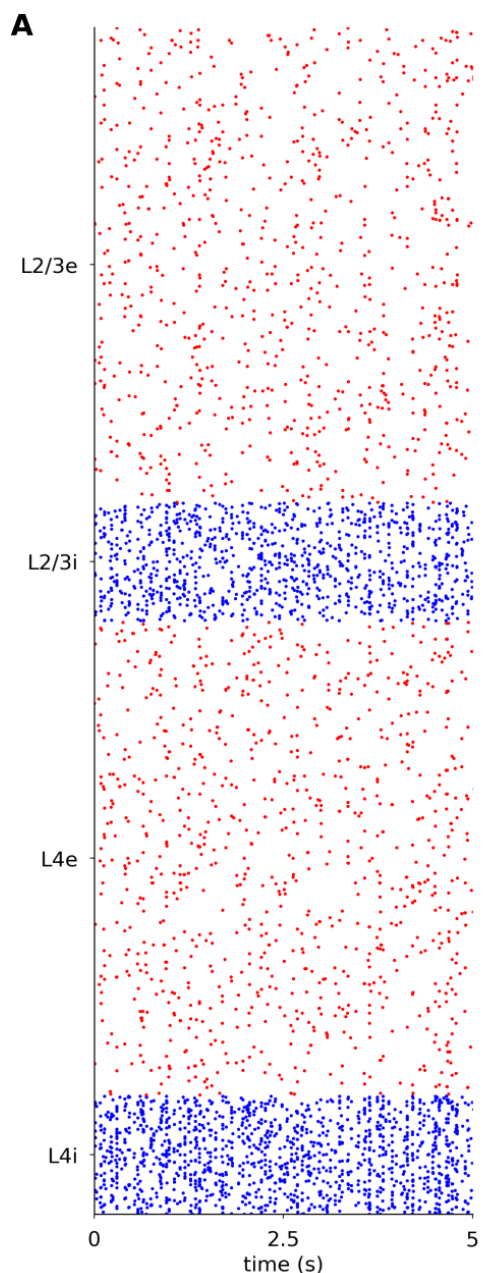

B

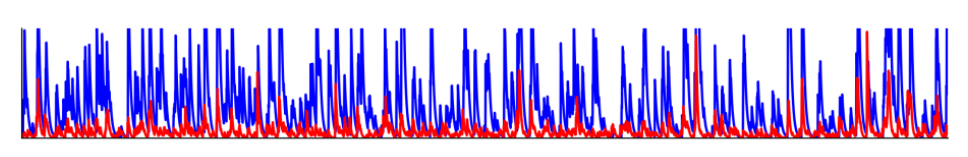

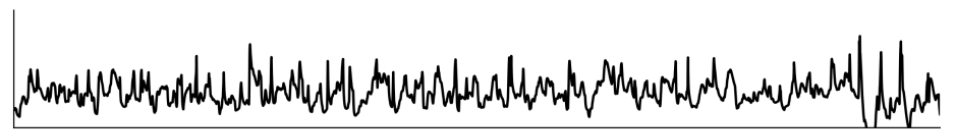

C
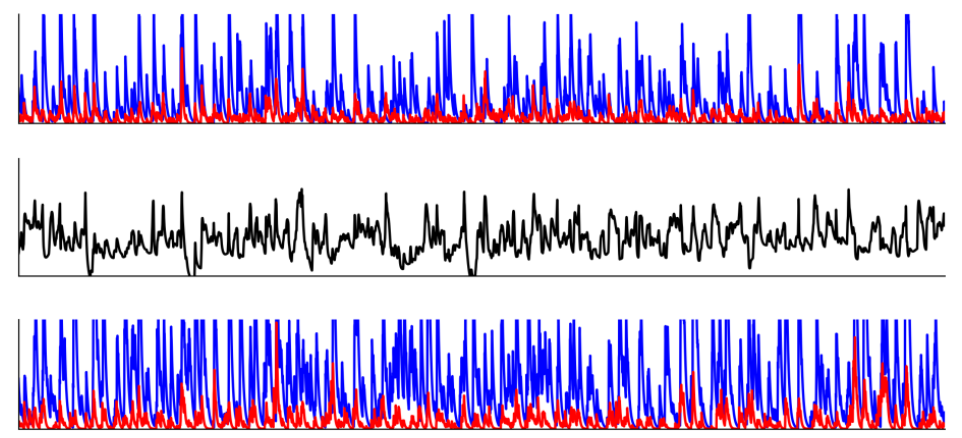

E
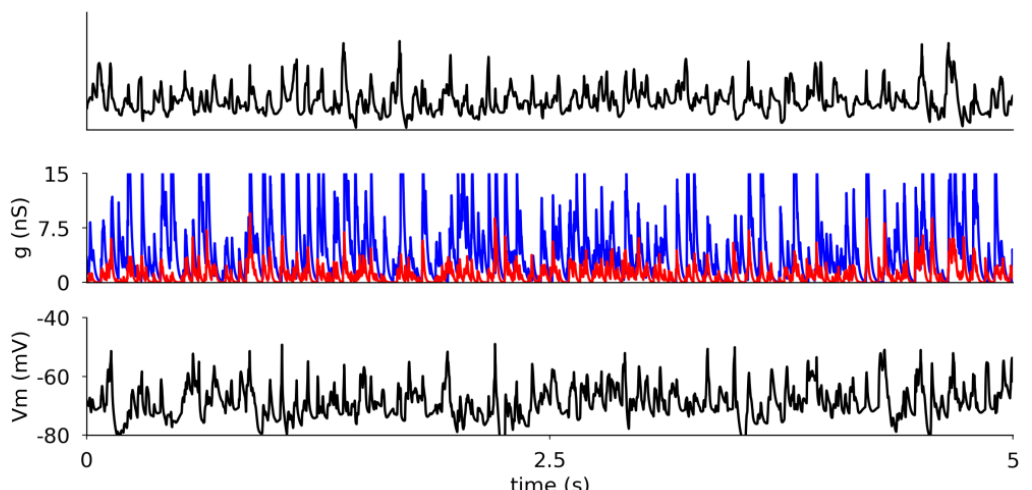

Figure 3: Spontaneous activity in modeled cortical layers. (A) Raster plot of spiking activity recorded for $5 \mathrm{~ms}$ of biological time in Layers 4 and 2/3 (from bottom to top; red: excitatory neurons, blue: inhibitory neurons). The relative number of spike trains shown corresponds to the relative number of neurons in the network (total of 400 neurons shown). (B-E) the membrane potential (black) and the excitatory (red) and inhibitory (blue) synaptic conductances of randomly selected neurons (example zoomed 1s time-course of the signals can be found here: L4 excitatory L4 inhibitory L2/3 excitatory L2/3 inhibitory ). (B) excitatory neuron in Layer 2/3; (C) inhibitory neuron in Layer 2/3 (C); (D) excitatory neuron in Layer 4; (E) inhibitory neuron in Layer 4. 
A
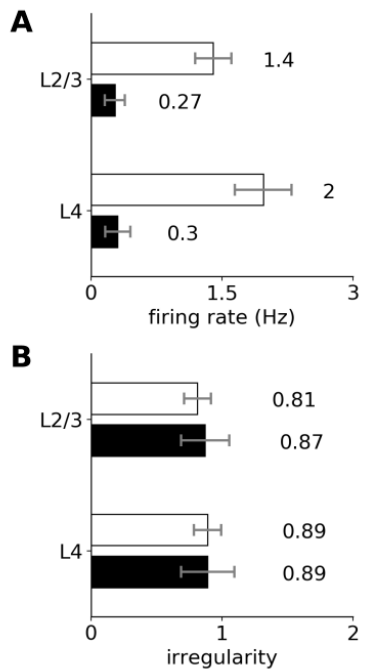

C

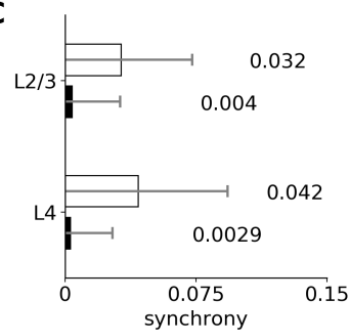

D

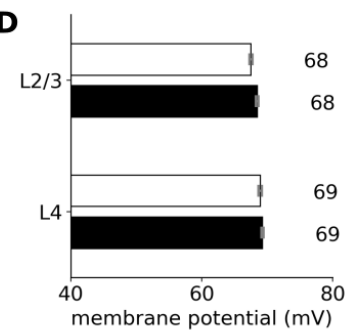

E

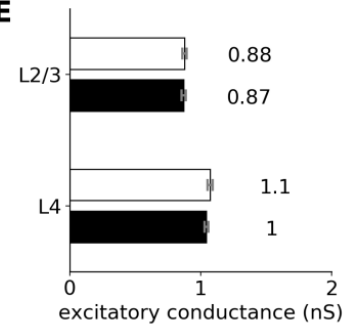

F

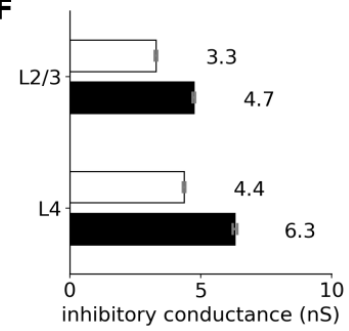

$\mathbf{G}$
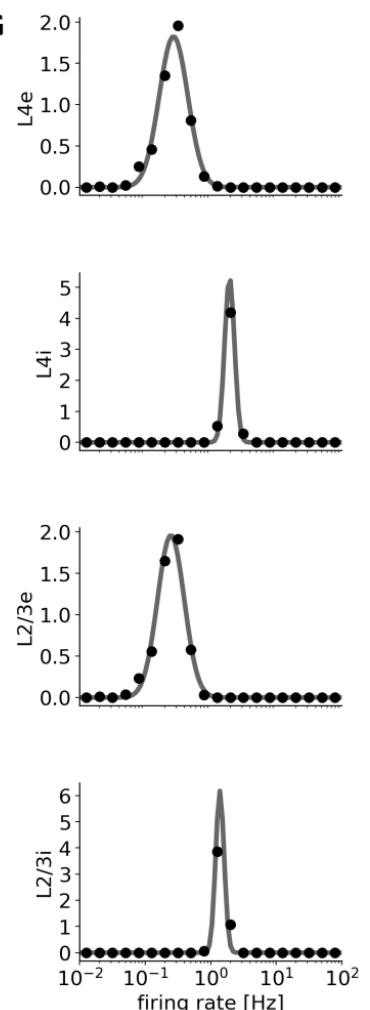
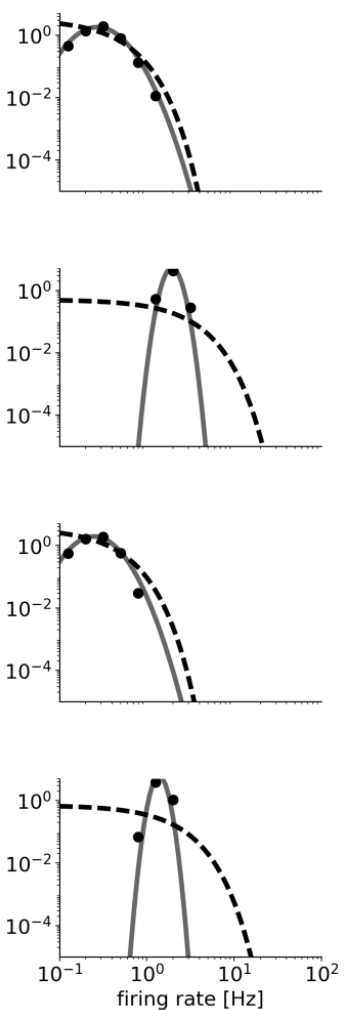

Figure 4: Statistics of spontaneous activity in the simulated network. (A-F) Six measures of spontaneous activity for excitatory (black bars) and inhibitory (white bars) neural populations in the two modeled cortical layers. Error bars report standard deviation. (A-C) Statistics of the spiking activity of the 4 modeled cortical populations based on spike trains recorded for $40 \mathrm{~s}$. (A) Mean single-unit firing rates. (B) Irregularity of single-unit spike trains quantified by the coefficient of variation of the inter-spike intervals. (C) Synchrony of multi-unit spiking activity quantified as the mean correlation coefficient between the PSTH (bin size $10 \mathrm{~ms}$ ) of all pairs of recorded neurons in a given population. (D-F) Mean values of intracellular variables: membrane potential (D); excitatory $(E)$ and inhibitory $(F)$ synaptic conductances. ( $G$ ) The distribution of firing rates (black points) in Layer 4 and 2/3 excitatory and inhibitory neurons is well fitted by a log-normal distribution with matching mean and variance (gray line; left-column). The log-normal distribution appears as a normal distribution on a semi-logarithmic scale. The data are better fit by a log-normal (gray line) than by an exponential (dashed line) distribution (right-column; log-log scale). 
We also investigated intracellular responses of the model neurons in the spontaneous state. The mean resting membrane potential of neurons is close to $-70 \mathrm{mV}$, as observed in in vivo cat $\mathrm{V} 1$ recordings (Monier et al. 2008). As shown in Figures $3 \mathrm{~B}$ and $4 \mathrm{FF}$, the excitatory synaptic conductances recorded in model neurons during spontaneous activity are low $(0.95 \mathrm{nS}$, averaged across all layers $)$ and close to the levels observed experimentally in cat V1 ( 1 nS; layer origin not known) (Monier et al. 2008). The inhibitory conductance during spontaneous activity was $5.1 \mathrm{nS}$ when averaged across all layers and neural types, also in a very good agreement with the value of $4.9 \mathrm{nS}$ reported in in vivo cat V1 recordings (Monier et al. 2008). The balance of excitation and inhibition during spontaneous condition in the model is thus in a very good agreement with in-vivo data in cat V1 (Monier et al. 2008). These levels of conductance are in stark contrast to those in most balanced random network models of spontaneous activity, in which the global synaptic conductance tends to be orders of magnitude higher (Vogels and Abbott 2005 Kumar et al. 2008). Overall the model exhibits a spontaneous state with balanced excitatory and inhibitory inputs well matched with cat V1 for both extra- and intracellular signals, without the need for any unrealistic external sources of variability. This represents an advance over previous models of $\mathrm{V} 1$ and over dedicated models of spontaneous activity, and enables a self-consistent exploration of stimulus-driven changes in variability in subsequent analysis (see Section 3.4).

\subsection{Responses to drifting sinusoidal gratings}

Figure 5 shows the responses of representative excitatory and inhibitory cells from both modeled cortical layers to multiple trials of optimally- and cross-oriented sinusoidal gratings of optimal spatial frequency, drifting at $2 \mathrm{~Hz}$. As shown in Figure $5 \mathrm{~A}$ (bottom panel), the membrane potential of the Layer 4 excitatory cell follows the sinusoidal modulation of the grating luminance-characteristic of Simple cells (Monier et al. 2008) - but remains largely unmodulated and below threshold during the cross-oriented condition. The sinusoidal shape of the membrane potential trace is the result of the excitatory and inhibitory synaptic conductances being mutually in anti-phase (Figure $5 \mathrm{~A}$ middle panel), as a result of the push-pull connectivity in model Layer 4. The trial-averaged excitatory and inhibitory synaptic conductances are low (mean value across recorded neurons $5.64 \mathrm{nS}$ and $29.4 \mathrm{nS}$ respectively), consistent with observations in cat V1 (Monier et al. 2008 Baudot et al. 2013). As a result of the sinusoidal sub-threshold dynamics, the spikes are generated in a periodic manner aligned to the upswings of the membrane potential, while the cross-oriented grating does not elicit significant depolarization despite the increase in overall input conductance, and thus the membrane potential remains below threshold and consequently no spikes are generated.

The spiking response of the inhibitory cells in Layer 4 (Figure 53 ) is similar, but the smaller number of incoming synapses (see Section 2.4.6) leads to slightly lower mean input conductances (mean value across recorded neurons; excitation: $5.0 \mathrm{nS}$, inhibition: $22.7 \mathrm{nS}$ ), while the stronger excitatory to inhibitory synapses (see Section 2.4.6) mean that the conductance balance is shifted towards stronger relative excitation during visual stimulation, thus leading to higher overall firing rates (see Section 3.2.1). Furthermore, due to the weaker inhibition, the cross-oriented grating still elicits slight depolarization of the membrane potential, leading to increased spiking activity, and resulting in small broadening of the orientation tuning curve, as further examined in Figure 6

Unlike the phasic response of Layer 4 cells, both excitatory and inhibitory cells in Layer $2 / 3$ show steady depo- 


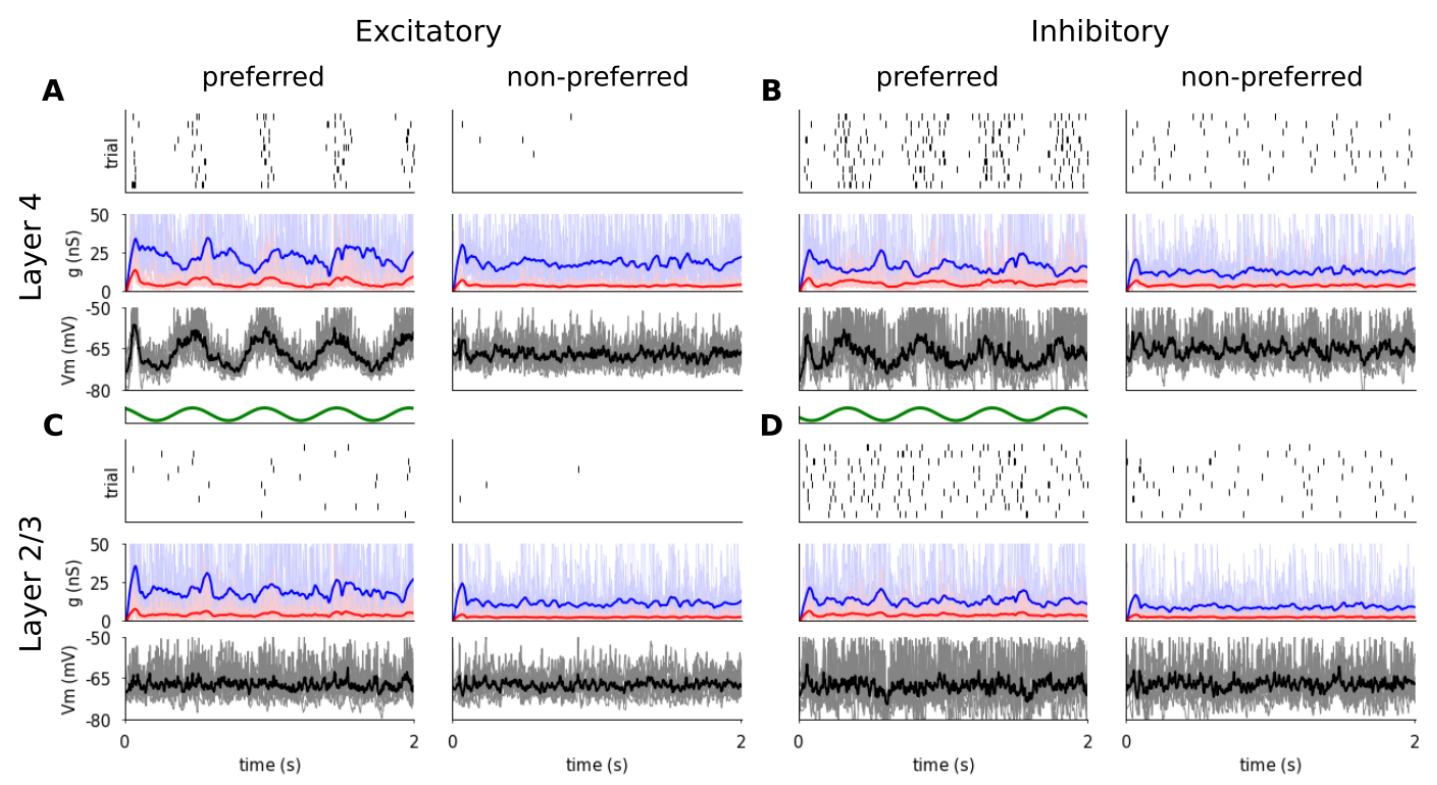

Figure 5: Responses of representative excitatory $(A, C)$ and inhibitory $(B, D)$ neurons from Layer $4(A, B)$ and Layer $2 / 3(C, D)$ to 10 trials of drifting gratings of optimal (left) and cross-oriented (right) orientations. For all neurons, the top panel is a spike raster plot, each line corresponding to a single trial. The middle panel shows the incoming excitatory (red) and inhibitory (blue) synaptic conductances and the bottom panel shows the membrane potential (thin lines: single trials; thick lines: mean). Stimulus onset is at time zero. Example single-trial $1 \mathrm{~s}$ time-course after stimulus onset at preferred orientation of the signals can be found here: L4 excitatory L4 inhibitory L2/3 excitatory L2/3 inhibitory

larization in response to an optimally orientated grating, similar to non-linear Complex cells (Figure 5 CD). The mean excitatory and inhibitory synaptic conductances are $3.7 \mathrm{nS}$ and $21.3 \mathrm{nS}$ respectively, within the range of conductance levels observed experimentally (Monier et al. 2008, Baudot et al. 2013).

\subsubsection{Orientation tuning of spike response}

We next examined the responses of the model to different orientations of the sinusoidal grating at two different contrasts. As illustrated in Figure 64, in both cortical layers most excitatory and inhibitory units had realistically shaped tuning curves (Alitto and Usrey 2004).

The mean tuning widths of excitatory and inhibitory neurons in Layer 4 and excitatory and inhibitory neurons in Layer $2 / 3$ measured as half-width-at-half-height $\left(\mathrm{HWHH}\right.$ ) were $23.8^{\circ}, 34.0^{\circ}, 24.6^{\circ}$ and $32.4^{\circ}$ respectively (see Figure 6C), which is within the range of tuning widths in cat V1 outlined by experimental studies (Nowak et al. 2008 Cardin et al. 2007; Finn et al. 2007). Even though some sub-groups of inhibitory neurons have been shown to be broadly tuned to orientation (Nowak et al. 2008), on average inhibitory neurons are well tuned (Cardin et al. 2007 Nowak et al. 2008), just as in our model. In both modeled layers inhibitory neurons were more broadly tuned (mean HWHH across all inhibitory neurons $32.4^{\circ}$ ) than the excitatory ones (mean $\mathrm{HWHH}$ across all excitatory neurons $24.1^{\circ}$ ), which is in a very good quantitative agreement with values measured by Nowak et al. (2008) (mean HWH of fast spiking neurons $31.9^{\circ}$, and regular spiking $23.4^{\circ}$ ).

It is important to note that because the HWHH measure does not take into account the unselective response 

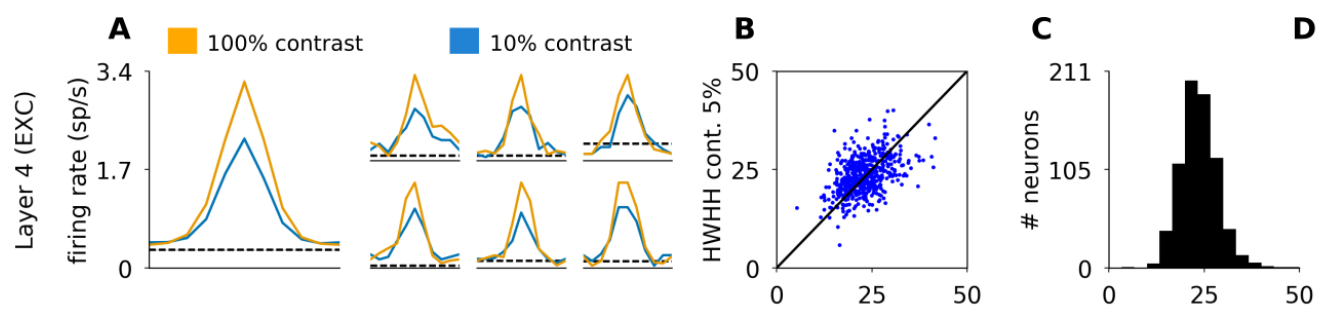

D
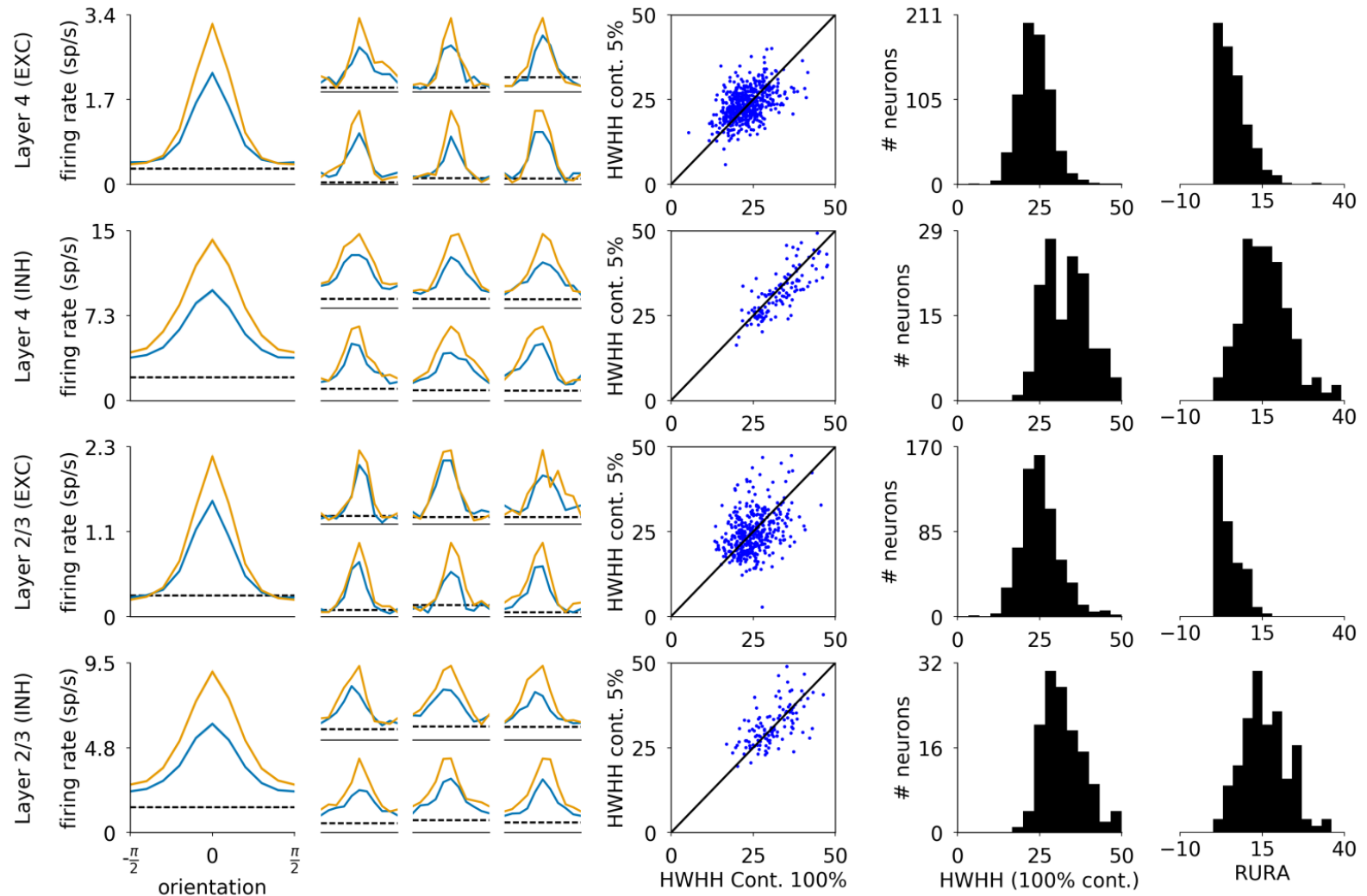

Figure 6: Orientation tuning properties of excitatory and inhibitory neurons in Layers 4 and 2/3. (A) Orientation tuning curves calculated as the mean firing rate of the neuron at the given orientation. The large tuning curves on the left represent the mean of centered orientation tuning curves across all the measured neurons of the given type. The smaller tuning curves on the right correspond to randomly selected single neurons. (B) Half-width-at-half-height $(\mathrm{HWHH})$ at $100 \%$ vs $10 \%$ contrast. (C) The distribution of $\mathrm{HWHH}$ measured at $100 \%$ contrast across the four modeled cortical populations. (D) The distribution of relative unselective response amplitude (RURA) measured at $100 \%$ contrast across the four modeled cortical populations. 
amplitude, cells that respond substantially above the spontaneous rate at all orientations, but where this unselective response is topped by a narrow peak, will yield low HWHH. To address this concern Nowak et al. (2008) also calculated the relative unselective response amplitude (RURA; see Methods), and showed that, while if viewed through the HWHH measure inhibitory neurons are only moderately more broadly tuned then excitatory ones, the RURA of inhibitory neurons is much broader. We have repeated this analysis in our model, and as can be seen in Figure $6 \mathrm{C}$ and $D$, the difference between excitatory and inhibitory neurons on the RURA measure is indeed much more pronounced ( $0.53 \%$ in excitatory vs $15.3 \%$ in inhibitory neurons), and in good quantitative agreement with values reported in Nowak et al. (2008) (mean for regularly spiking neurons $2.5 \%$ and fast spiking neurons neurons $18.1 \%$ ).

As evidenced by the mean and single neuron orientation tuning curves shown in 64 , most excitatory and inhibitory units in the model cortical Layer 4 and 2/3 exhibit contrast invariance of orientation tuning width, which is further confirmed by comparing the HWHH of the tuning curves at low and high contrast (Figure 63 : most points lie close to the identity line). On average we observe very minor broadening of the tuning curves at high contrast, and it is more pronounced in inhibitory neurons: the mean $\mathrm{HWHH}$ differences between low and high contrasts is $0.8^{\circ}$ (excitatory, Layer 4), $1.51^{\circ}$ (inhibitory, Layer 4), $0.14^{\circ}$ (excitatory, Layer $2 / 3$ ), and $0.83^{\circ}$ (inhibitory, Layer $2 / 3$ ). Such minor broadening has been observed experimentally in Simple cells in cat V1 $\left(0.3^{\circ}\right.$; layer origin nor neural type not known Finn et al. 2007).

Overall, the orientation tuning in our model is in very good qualitative and quantitative agreement with experimental data, with both excitatory and inhibitory neurons exhibiting sharp and contrast-invariant orientation tuning across all modeled layers, in contrast to many previous modeling studies that relied on untuned inhibition, or broad contrastdependent inhibition (Troyer et al. 1998 Lauritzen and Miller 2003 Chariker et al. 2016).

\subsubsection{Orientation tuning of sub-threshold signals}

To better understand the mechanisms of orientation tuning in the model, we investigated the tuning of the membrane potential and the excitatory and inhibitory synaptic conductances. An essential mechanism responsible for orientation tuning in this model is the push-pull connectivity bias in Layer 4. In theory, a cell with a pure push-pull mechanism with perfectly balanced excitation and inhibition should exhibit purely linear (Simple cell) behavior, where orientation tuning is solely driven by the luminance modulation of the drifting grating, and the mean membrane potential should remain at resting (spontaneous) level at all orientations. In order to assess to what extent this idealized scheme is true in this model, we have examined the DC (F0) and first harmonic (F1) components of the analog signals, and plotted the mean orientation tuning curves across the different neural types and layers in Figure 7

The orientation tuning of membrane potential in Layer 4 (in both excitatory and inhibitory neurons) is dominated by the $\mathrm{F} 1$ component, in line with the presence of strong push-pull mechanisms in this model layer (Figure 7 B). Layer 4 cells also have significant tuning of the mean membrane potential, but of smaller magnitude (Figure 7 A). The F0 components of the membrane potential show noticeable broadening at higher contrast, consistent with observations by Finn et al. (2007) that, unlike the spiking response, the orientation tuning width of the mean of membrane potential is not contrast independent. In Layer 2/3 the magnitude of the $\mathrm{Vm}$ components is reversed: the magnitude of the 


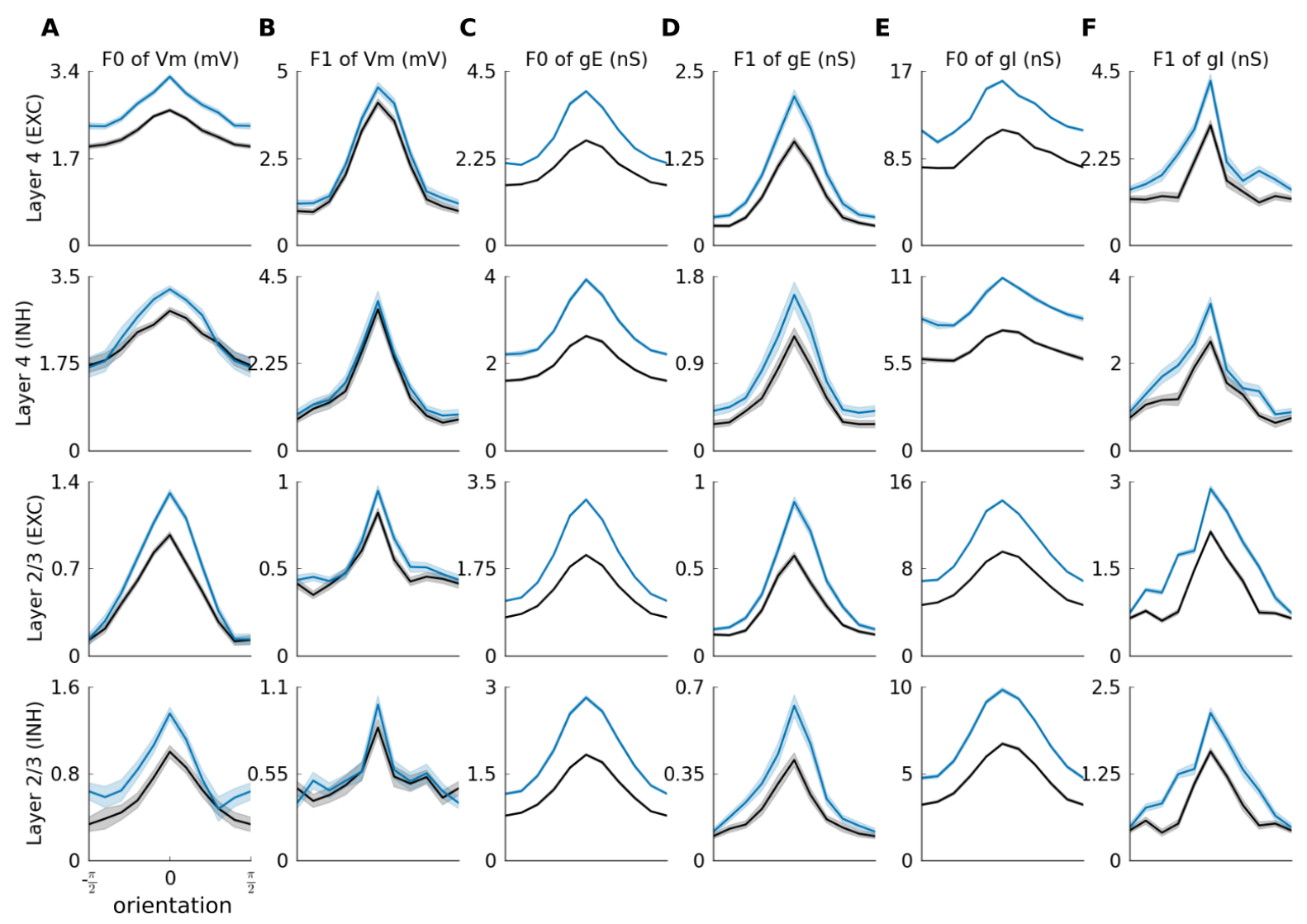

Figure 7: Orientation tuning of membrane potential and excitatory and inhibitory conductances of excitatory and inhibitory neurons in Layer 4 and 2/3. Each row shows a different layer and neural type, see labels. Orientation tuning curves of: (A) F0 component of membrane potential; (B) F1 component of membrane potential; (C) F0 component of excitatory synaptic conductance; (D) F1 component of excitatory synaptic conductance; (E) F0 component of inhibitory synaptic conductance; (F) F1 component of inhibitory synaptic conductance. Low contrast: 10\% (blue line), high contrast: 100\% (black line). 
F0 component of the membrane potential is stronger than the F1 component. This is consistent with the lack of phase specific connectivity in this model layer, and explains the predominance of Complex cells in this layer, as will be further elaborated in Figure 8. Furthermore, for excitatory cells in Layer 2/3, unlike in those in Layer 4, the F0 of the membrane potential exhibits contrast invariance of tuning width.

How does the interplay between excitation and inhibition lead to the observed membrane potential tuning characteristics? To answer this question, in Figure $7 \mathrm{C}-\mathrm{F}$ we plot the orientation tuning curves of the F0 and F1 components of excitatory (C,D) and inhibitory (E,F) synaptic conductances. In Layer 4, the most obvious difference is that in comparison to the tuning of membrane potential, the F0 component of both excitatory and inhibitory conductances dominates the F1 component. The weaker F0 component of the membrane potential in Layer 4 neurons is thus due to the cancellation between the excitatory and inhibitory F0 components, while the F1 component of the membrane potential orientation tuning remains strong, as such cancellation between excitatory and inhibitory conductances does not occur due to the half period phase shift between them (see Figure 5 A,B). In Layer 2/3 both F0 and F1 of excitatory and inhibitory conductances in both excitatory and inhibitory neurons are well tuned, but the F0 components dominate in magnitude the $\mathrm{F} 1$ components both for excitatory and inhibitory conductances and in both neural types.

Overall we observe that for all layers and cell types both F0 and F1 components of membrane potential and conductances exhibit orientation tuning of varying degree, consistent with observations made by Anderson et al. (2000). The contrast invariance in orientation tuning arises as a complex interplay between excitation and inhibition, differs between layers and neural types, and in the model Layer 4 is consistent with engagement of a push-pull mechanism (Troyer et al. 1998). However, additional enhancement of activity is present due to the recurrent facilitatory dynamics among neurons of similar orientation, that goes beyond the predictions of the push-pull model, as evidenced by the presence of tuning in the F0 components of the membrane potential of layer 4 neurons.

\subsubsection{Simple and Complex cell types}

Next we examined the classification of the cells into Simple and Complex. In line with the behavior of the representative model neurons shown in Figure 5 the modulation ratio computed from the PSTH of most neurons in Layer 4 is greater than one, which classifies them as Simple cells (see Figure 84). The same measure for most neurons in Layer 2/3 is less than one, classifying them as Complex cells (see Figure 84). This laminar bias towards different modulation ratios is in line with previous experimental evidence (Hirsch and Martinez 2006). When pooled across the two layers the histogram of modulation ratios forms a bimodal distribution (see Figure $8 \mathrm{~A}$ ), as observed experimentally (Priebe et al. 2004).

Unlike for the PSTH, the modulation ratios computed from the membrane potential Vm (with the resting potential subtracted) when pooled across the two modeled cortical layers form a unimodal distribution (see Figure $8 \mathrm{~B}$ ), in line with experimental evidence in cat (Priebe et al. 2004). However, the range of modulation ratios of $\mathrm{Vm}$ is higher in the model compared to cat $\mathrm{V} 1$, due to a greater number of cells that behave nearly linearly in $\mathrm{Vm}$. When the modulation ratios calculated from PSTH and membrane potential are plotted against each other (Figure 8 F), a characteristic hyperbolic relationship is revealed, in line with the observations of Priebe et al. (2004). However, when analyzing 

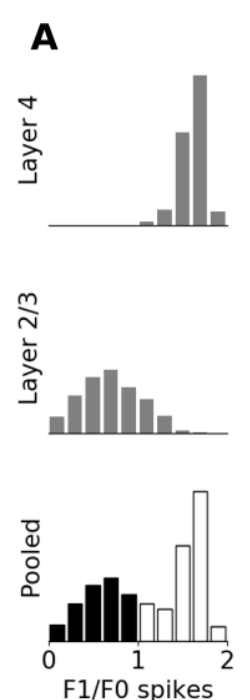

B
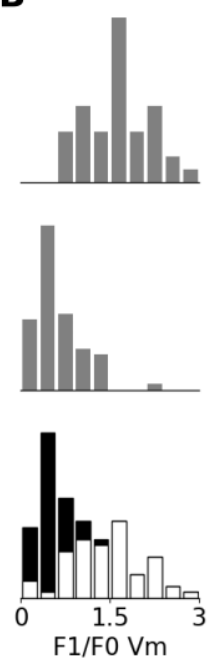

C
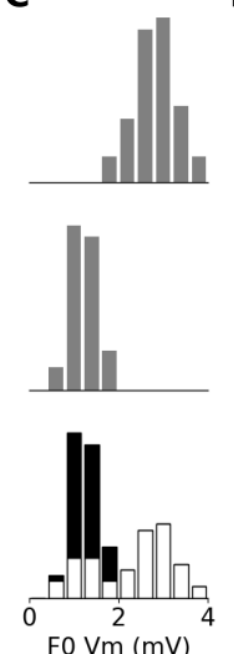

D

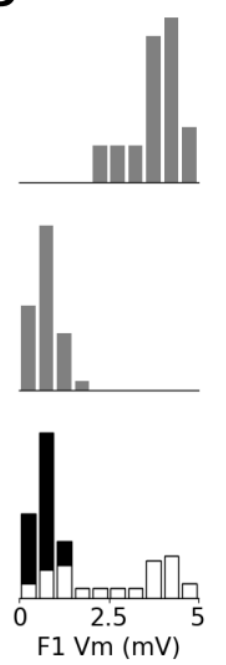

$\mathbf{E}$

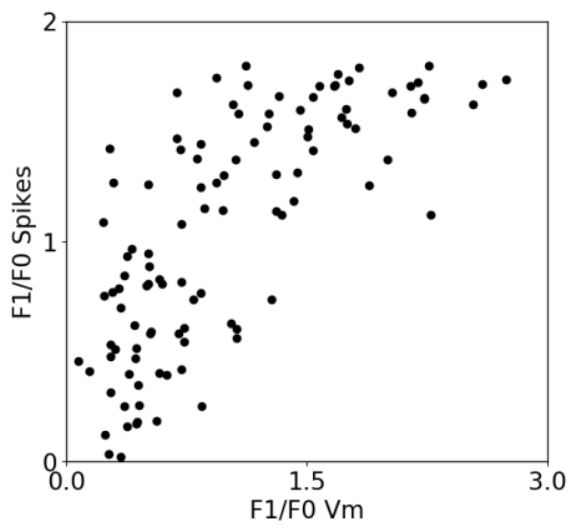

Figure 8: Modulation ratio of excitatory cells in the model. (A) Histogram of modulation ratios calculated from the PSTH over recorded excitatory cells in Layer 4 (first row), Layer 2/3 (second row) and pooled across both layers. (B) as in (A) but modulation ratio calculated from the membrane potential after subtraction of background level. (C) As in A but the histograms are of the absolute value of the F0 component of the membrane potential. (D) As in $\mathrm{C}$ but for the $\mathrm{F} 1$ component of the membrane potential. (E) The relationship between the modulation ratio calculated from PSTH and from membrane potential. In the pooled plots cells classified as Simple are marked white and as Complex are marked black.

the F0 and F1 components of the membrane potential individually, some differences from the experimental data are revealed.

The main discrepancy is that the distributions of the F0 and F1 components of membrane potential 8 C,D) are narrower in both modeled layers, than experimentally observed (Priebe et al. 2004), which in the case of the F1 component of membrane potential leads to a bimodal distribution when pooled across the two modeled layers, unlike the data of Priebe et al. (2004). It should be noted though, that in that study, the histogram showing the F1 component of membrane potential, more than $90 \%$ of data is in the first 3 bars, and so it is not possible to say if 'zooming' into the lower range of values, which encompasses an absolute majority of cells, might not reveal a separation between Simple and Complex cells in this measure. Furthermore, the overall lower range of values for the F0 and F1 measures in the model (compared to cat data) that contributes to the bimodality after pooling across layers could be due to intrinsic regularities present in the model, such as identical physiological parameters among all cells of the same cell type, or limited variability of the ratio of afferent and recurrent inputs to model Layer 4 neurons, or completely phase-non-specific connectivity from Layer 4 to $2 / 3$. These artificial regularities introduced in the model could be exaggerating the differences between the two functional neural types. Unfortunately, the values of these parameters in cat V1 have not yet been investigated, but it is reasonable to expect some variation, and introducing such variability in the model could thus rectify these discrepancies. 


\subsection{Size tuning}

We have also examined the most well studied manifestation of contextual modulation, size tuning, whereby one presents a drifting sine grating confined to an aperture of increasing diameter, and records the response as a function of the aperture diameter. Figure 9 shows the size tuning properties of excitatory cells in both modeled cortical layers. Most excitatory neurons in both cortical layers show surround suppression (Figure 9A-D), however we observe a diversity of tuning patterns. In the first row of Figure 9 we can see an example of Layer 4 cells with prototypical size tuning properties, including the expansion of facilitation diameter at low contrast (Wang et al. 2009: Tailby et al. 2007). On the other hand, in the second row of Figure 9 we show an example of a Layer 4 cell that exhibits only weak suppression and a minor contrast dependent shift of facilitation diameter. Neurons in Layer $2 / 3$ also express classical size tuning effects as exemplified in the third row of Figure 9

The degree of the suppression varies widely in both cortical layers (Figure 9D). Overall the suppression is stronger in Layer 2/3 (mean suppression index (SI) at high contrast: $0.34 \pm 0.009$ ) than in Layer 4 (mean SI at high contrast: $0.24 \pm 0.012)$. These values are well within the range observed experimentally in cat V1 (i.e. 0.16 (Tailby et al. 2007), 0.35 (Okamoto et al. 2009), 0.44 (Wang et al. 2009) and 0.47 (Akasaki et al. 2002); cells pooled across all cortical layers). Stronger suppression in Layer 2/3 than in Layer 4 was observed in three studies (Okamoto et al. 2009. Akasaki et al. 2002, Jones et al. 2000) while Wang et al. (2009) did not observe a statistically significant difference in SI between Layer 4 and 2/3. Finally, in line with Wang et al. (2009), we observe a decrease of suppression at low contrast (mean SI at low contrast: $0.23 \pm 0.012$ Layer 4 and $0.28 \pm 0.016$ Layer 2/3).

At high contrast, in Layer 4 neurons we observe maximum facilitation diameter (MFD) of $2.0^{\circ} \pm 0.04^{\circ}$ and in Layer $2 / 3$ of $1.88^{\circ} \pm 0.019^{\circ}$ (Figure $9 \mathrm{G}$ ). It is difficult to precisely compare these numbers to experimental data, as RF size in cat V1 grows with its foveal eccentricity (Wilson and Sherman 1976), but we are not aware of any study that reports eccentricity-dependent data on size tuning. Studies have reported a broad range of mean MFD values $\left(2.9^{\circ}\right.$ (Sengpiel et al. 1997), 3.5 (Jones et al. 2000), $6^{\circ}$ (Tailby et al. 2007)). Values observed in our study are slightly below this range, which is, however, consistent with the fact that all these studies recorded neurons at (on average) larger eccentricities than the $3^{\circ}-$ a value on which our model is centered (see Methodology). We, however, do observe important trends consistent with the experimental data. First, we observe increase of the the MFD at lower contrast (Figure 9G; mean MFD at low contrast: $2.39^{\circ} \pm 0.036$ Layer 4 and $2.18^{\circ} \pm 0.089$ Layer $2 / 3$ ), although this change of $\sim 20 \%$ is slightly lower than in Wang et al. (2009) and Tailby et al. (2007) who found increases of $33 \%$ and $36 \%$ respectively in cat V1. Second, we observe smaller MRF in Layer 2/3 than in Layer 4 (see above data) in line with observations in Jones et al. (2000). Finally, we find that both excitatory and inhibitory conductances are size tuned at both low and high contrast and in both simulated cortical layers (Figure $9 \mathrm{H}$ ), in line with experimental evidence (Anderson et al. 2001 Ozeki et al. 2009).

A more recent finding regarding surround suppression is that many neurons do not exhibit monotonic suppression after reaching their classical RF size: after a certain diameter their responses partially recover from the suppression (Wang et al. 2009). This phenomenon has been named counter-suppression. We observe this in a substantial subset of model neurons across both modeled layers (Figure $9 \mathrm{E}$ ). When quantifying the magnitude of this counter-suppression 

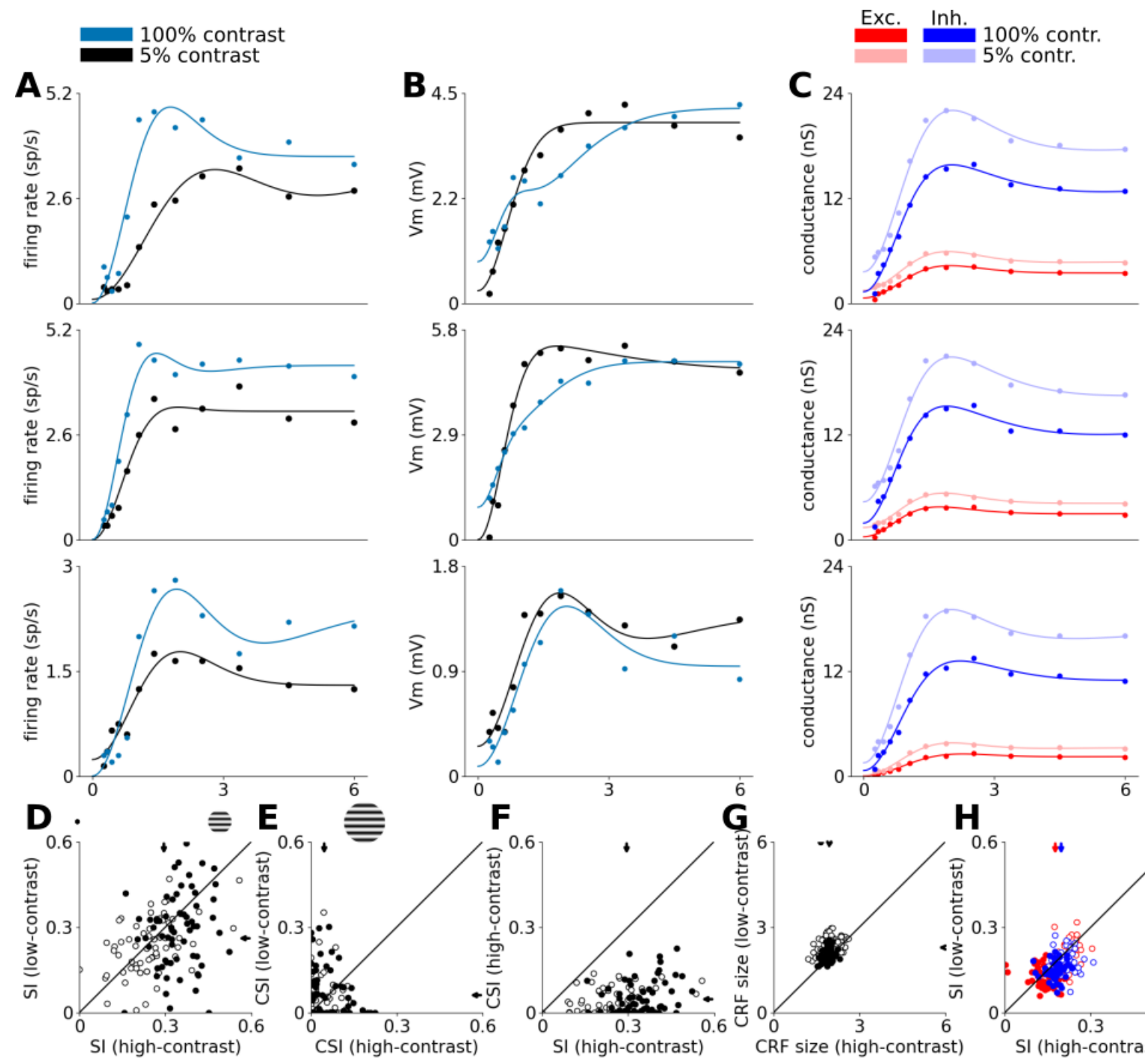

CRF size (high-contrast)
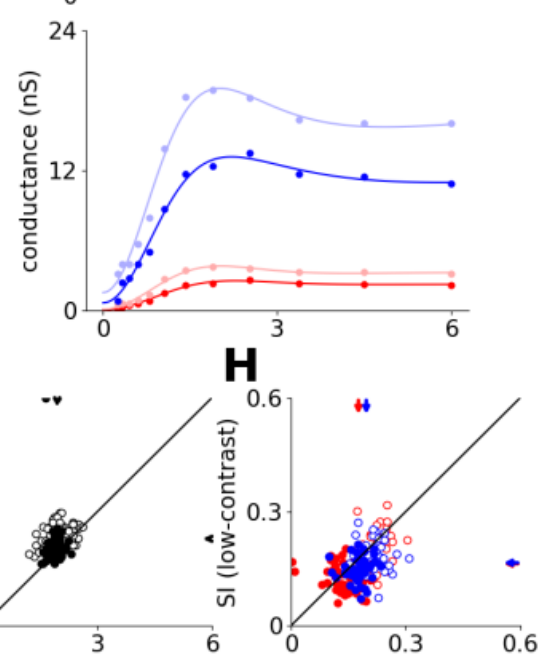

Figure 9: Size tuning in excitatory neurons. The first three rows show size tuning in three example model neurons. Top row is a typical Layer 4 cell with strong size tuning, second row shows an example of Layer 4 cells that does not exhibit size tuning, and the third row shows a typical Layer $2 / 3$ cell. (A) The size tuning curve of trial-averaged spiking responses. Dots are measured responses, line is fitted size tuning curve. (B) The size tuning curve of trial-averaged mean membrane potential. (C) The size tuning curves of trial-averaged mean excitatory (red) and inhibitory (blue) synaptic conductances. Data shown for low and high contrast conditions: (A-B) low-contrast (10\%) condition is blue, high-contrast $(100 \%)$ condition is black; (C) lowcontrast $(10 \%)$ condition is un-saturated, high-contrast $(100 \%)$ condition is saturated; (D-H) Scatterplots of size-tuning measures. Arrows mark means after pooling across layers. White (empty in $\mathrm{H}$ ) dots correspond to individual Layer 4 neurons and black (full in $\mathrm{H}$ ) dots correspond to Layer 2/3 neurons. (D) The suppression index of spike responses at low vs. high contrast. (E) The counter-suppression index of spike responses at low vs. high contrast. (F) The suppression index vs. the counter-suppression index at high contrast. (G) the maximum facilitation diameter at low vs. high contrast. (H) The suppression index of excitatory (red) and inhibitory (blue) synaptic conductances at low vs. high contrast. 
as the CSI index, Wang et al. (2009) found that it tends to be stronger at low contrast than at high contrast, and that the suppresion index $\mathrm{SI}$ is almost always stronger than the CSI. In our model we find that on average the CSI index at low contrast is moderately stronger (mean CSI at low contrast: $0.05 \pm 0.008$ Layer 4 and $0.07 \pm 0.01$ Layer 2/3) than at high contrast (mean CSI at low contrast: $0.04 \pm 0.005$ Layer 4 and $0.052 \pm 0.007$ Layer 2/3), which is qualitatively in line with Wang et al. (2009), although we observe a less robust effect in the model, i.e. a smaller proportion of neurons follow the trend, than in cat (Figure $9 \mathrm{E}$ ). It should be, however, noted that the limited area of the simulated cortical network likely prevents the full build-up of the counter-suppression effects, and is the likely reason for the less robust expression of this long-range phenomenon in the model vs. cat data. We do also observe the stronger SI over CSI in an absolute majority of model neurons, in very good accordance with Wang et al. (2009) (Figure 9F). Finally, it should be pointed out, that we observe the onset of counter-suppression already at lower diameters $\left(<6^{\circ}\right)$, whereby Wang et al. (2009) note that they usually observe the counter suppression only at diameters greater than $10^{\circ}$ (no quantitative data provided). However, as we have explained in previous paragraph, it is reasonable to assume that, just like RF size, the diameter of the counter-suppression onset will be lower at the lower eccentricities (compared to Wang et al. 2009) at which our model neurons are located.

\subsection{Trial-to-trial variability: response to natural images with simulated eye-movements}

Next we probed the model with a stimulus consisting of a natural scene animated with simulated eye-movements, as described in the intra-cellular study of Baudot et al. (2013). As can be seen in Figure $10 \beta$, the natural image stimulus elicits a highly repeatable response, both at the level of spikes and at the level of sub-threshold responses, unlike the response to presentation of a drifting grating which is only locked to the slow temporal frequency of the luminance modulation, in accordance with findings by Baudot et al. (2013) in cat V1.

To further investigate the response precision and reliability of the model neurons we computed the cross-correlation between trials both of the spiking responses and of the membrane potential responses. The reliability is given by the peak amplitude of the cross-correlation $(a)$ at time zero, and the temporal precision by the standard deviation of the Gaussian fit $(\sigma)$ (Baudot et al. 2013). As shown in Figure 10, D, for the spiking response (top), both for model Layer 4 and Layer 2/3 neurons, the cross-correlation has a higher peak and is narrower for the natural-image-with-eyemovement (NI) stimulus (Layer 4: $a=0.1, \sigma=6.7$; Layer 2/3: $a=0.03, \sigma=8.2$ ), than for drifting sinusoidal gratings (Layer 4: $a=0.05, \sigma=61$; Layer 2/3: $a=0.0079, \sigma=60)$, in line with the experimental results in Baudot et al. (2013) (see Figure $10 \mathrm{~F}$ ).

For the membrane potential (Figure 10C,D bottom) the same relationship holds except that for Layer 4 neurons the peak (and thus the reliability) for gratings is slightly lower than for natural images (Layer 4 natural images: $a=0.37$, $\sigma=11$; Layer $2 / 3$ natural images: $a=0.12, \sigma=8.7$; Layer 4 gratings: $a=0.4, \sigma=91$; Layer $2 / 3$ gratings: $a=0.02, \sigma=46)$, unlike the observations in Baudot et al. (2013) (Figure 10F), where the reliability is higher for the NI stimulus for both spikes and membrane potential. However, note that in Baudot et al. (2013) the results are pooled across neurons of all layers, and the same treatment of our data produces higher reliability for natural images also in the model, in line with Baudot et al. (2013) (Figure 10E). Therefore this can be considered a prediction of our model, specifically that 
the reliability of membrane potential responses for Simple (or Layer 4) cells alone is higher for drifting gratings than for NI stimuli.

Furthermore, Baudot et al. (2013) found that relative to the trial-to-trial variability of membrane potential during spontaneous activity, the variability of Vm during DG stimulation increases, while it decreases during NI stimulation (Figure $10 \mathrm{G}$ ). We have performed the same analysis on the model, the results of which are shown in Figure 10 ( $\mathrm{HI})$. In both model layers we observe an increase of stimulus-locked variance (calculated as the inverse of the stimulus-locked time-averaged standard deviation across trials, and expressed as a percentage relative to the value for ongoing activity) for DG (97 $\pm 0.68 \%$ in Layer 4 and $96 \pm 0.23 \%$ in Layer $2 / 3$ ) but a decrease of stimulus-locked variance for the $\mathrm{NI}$ condition (122 $\pm 0.99 \%$ in Layer 4 and $104 \pm 0.35 \%$ in Layer 2/3), in line with Baudot et al. (2013).

The lack of direct external noise injected into cortical model neurons was crucial for achieving the results presented here. The trial-to-trial variability in the model is primarily shaped by the intra-cortical interactions during both spontaneous and stimulus driven activity. This is an advance over a recent model of a V1 column that explained the precision and reliability changes between the grating and naturalistic stimuli at the spiking level but did not reproduce the stimulus-dependent variability effect at the level of membrane potential (Kremkow et al. 2016). This was due to the inclusion of substantial random spiking input directly into cortical neurons, which induced substantial stimulusindependent variability of the membrane potential, overriding the internally generated components. These differences point out the importance of simultaneously capturing within a single model the cortical processing under multiple stimulation conditions and at different levels of signal integration, to be able to fully describe its operation.

The variability in our model can be attributed to two principal sources: the stochastic nature of thalamo-cortical and cortico-cortical connectivity wiring-pattern generation, and the intrinsic thalamic variability (simulated as a whitenoise source). We have shown that these sources are sufficient to explain variability in a number of measures of cortical function (Fig 4 and 10). But it is reasonable to expect that additional sources of variability exist. One such major source of variability not considered here is the stochastic nature of information transmission at synapses (Stratford et al. 1996 Allen and Stevens 1994). Another key factor reducing variability in the model is the regularity in the generation of its architecture. For example we made the approximations of fixed size, spatial frequency and aspect ratio of RF templates of Layer 4 V1 neurons. We also modeled the ON and OFF channels as being of equal strength, even though recent electrophysiological investigations revealed the dominance of the OFF pathway and systematic variations of the ON-OFF pattern of RF following the orientation maps (Kremkow et al. 2014 Wang et al. 2014). All these structural parameters are known to have substantial biological variability (Jones and Palmer 1987) Cardin et al. 2007 Ringach 2004). Unfortunately, quantitative estimates of these various sources of variability are not currently available. Our modeling paradigm could be used in the future to formulate predictions for these values by evaluating which additional sources of variability and of what magnitude could account for the experimental observations.

\subsection{Knowledge integration}

The central goal of the model presented here was to achieve knowledge integration that goes beyond what has been accomplished in previous computational studies of $\mathrm{V} 1$. This of course does not mean that we have managed to 
A
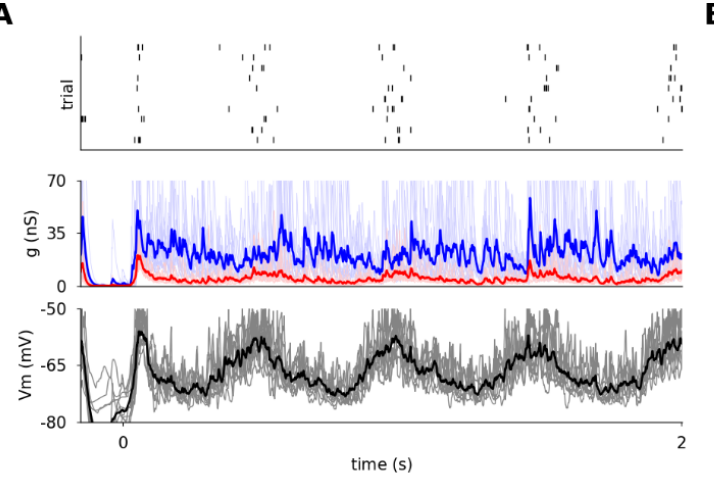

C

Layer 4
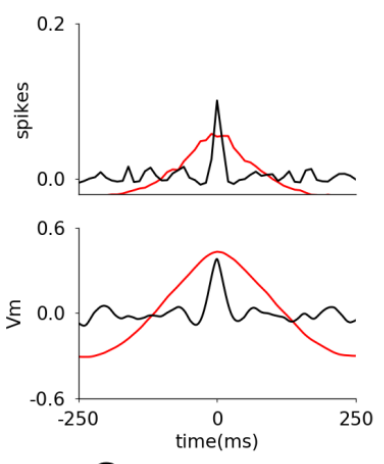

G

Data
D

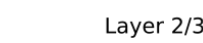

H

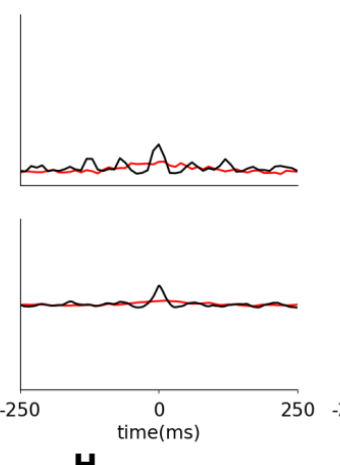

Layer 4
B
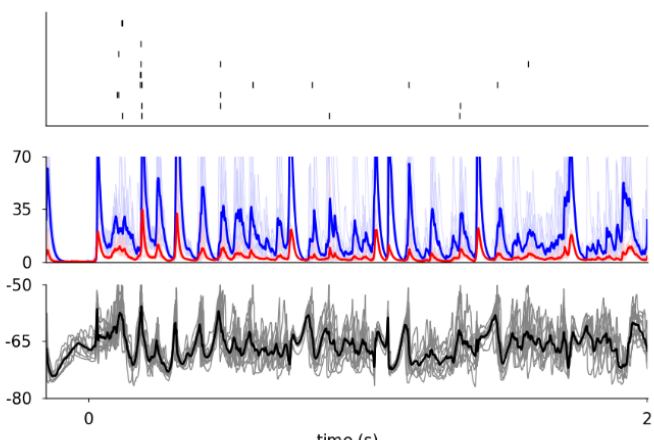

time (s)

$\mathbf{F}$

F $\quad$ Data (cat)

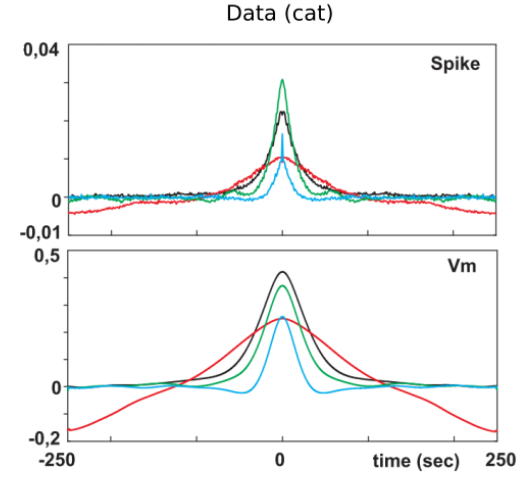

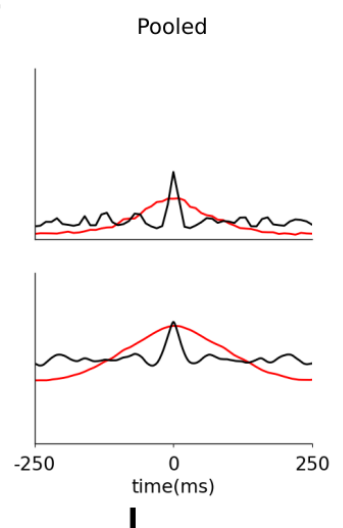

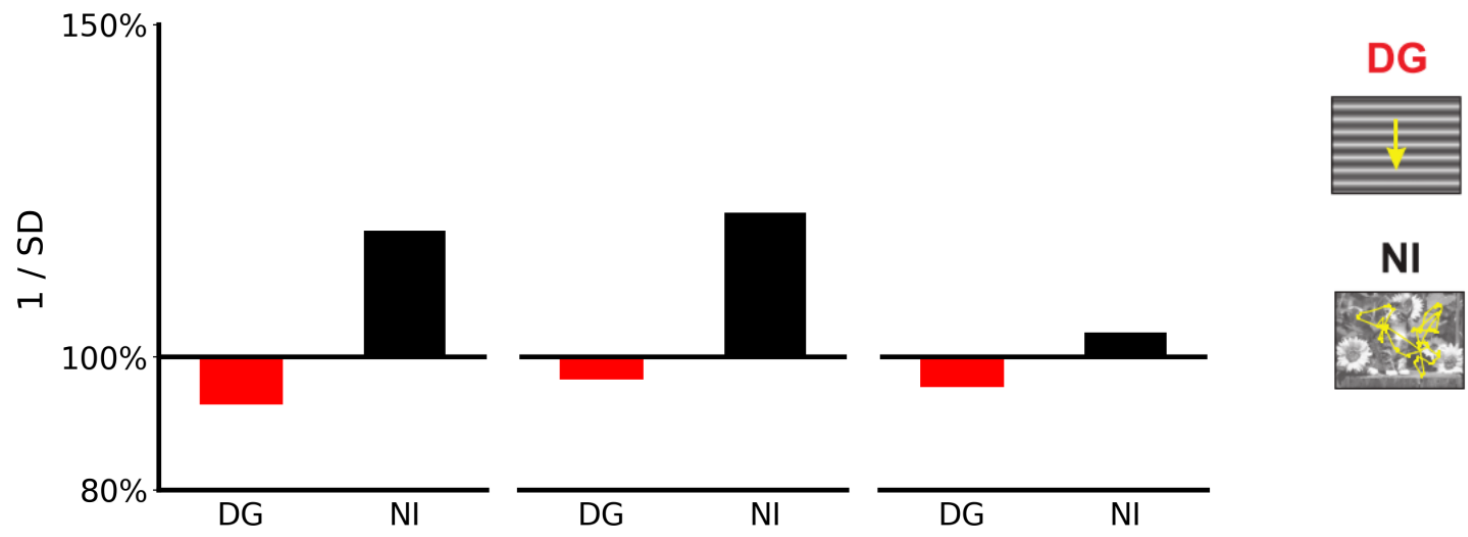

Figure 10: Trial to trial reliability of responses to a natural image with simulated eye movements (NI) and to drifting sinusoidal gratings (DG). (A) The response of a randomly sampled excitatory neuron in model Layer 4 to the DG stimulus. The top panel shows the spike raster histogram, the middle panel shows the incoming synaptic excitatory (red) and inhibitory (blue) conductances. The bottom panel shows the membrane potential. The thick lines show the mean of the analog signals over the 10 trials. (B) As (A), but for the NI stimulus. Zoomed single-trial 1s time-course after stimulus onset version can be found here (C-F) Cross-correlation across trials for spikes (top) and subthreshold membrane potential activity (bottom), in response to DG and NI stimuli. The reliability is given by the peak amplitude at time zero, and the temporal precision by the standard deviation of the Gaussian fit to the cross-correlation. (C) Results averaged across all recorded excitatory neurons in model Layer 4. (D) Results averaged across all recorded excitatory neurons in model Layer 2/3. (E) Results averaged across all recorded neurons pooled across the two model layers. (F) The experimental data from Baudot et al. (2013). Note that in that study two additional stimuli were also presented (dense noise : blue line; grating with simulated eye-movements : green line) that we have not used in this study. (G-I) The inverse of the stimulus-locked time-averaged standard deviation of membrane potential (1/SD) across trials, averaged across cells, relative to the value for ongoing activity. (G) Experimental results in cat (Baudot et al. 2013). (H) Results averaged across all recorded excitatory neurons in model Layer 4. (I) Results averaged across all recorded excitatory neurons in model Layer 2/3. 
Table 2: Validation of visual cortex models with respect to experimentally observed functional properties. A green tick means that the model gives a qualitatively good match to experimental findings. A red cross means that the model gives a response which is qualitatively different from experimental findings. A red open circle means that the model does not incorporate neural structures necessary for this feature to be meaningfully tested. Empty spaces mean that the model was not tested for the property, and it is not possible with certainty to conclude from the corresponding paper that the model does or does not match corresponding experimental data. 
bioRxiv preprint doi: https://doi.org/10.1101/416156; this version posted November 8, 2019. The copyright holder for this preprint (which was not certified by peer review) is the author/funder, who has granted bioRxiv a license to display the preprint in perpetuity. It is made available under aCC-BY 4.0 International license.

demonstrate every property of V1 that has been successfully modeled in the past, but rather that the model was tested by the broadest range of visual stimulation protocols and reproduces the greatest range of experimentally demonstrated properties of V1 computation to date. To demonstrate that this goal has been met, we present in this section a crosscomparison of the presented model with the most prominent integrative models of $\mathrm{V} 1$ to date. We restrict this comparison to models of cat and macaque $\mathrm{V} 1$, as the two species represent the two major animal models in vision while their anatomical and functional similarity allows for straightforward comparison. Other models of V1 relevant to this study that do not meet this criterion are commented on in the Discussion. Table 2 provides an overview of the various V1 features that have been successfully modeled in the different previous integrative models of V1 function and in the present model. More in-depth comparison of the selected models follows.

Somers et al. (1998) investigated a topologically-organized model of V1, which incorporated excitatory connections that dominated at short distances, and inhibitory ones that dominated at medium distances. Evidence for such direct medium-range inhibitory connections is lacking (Stepanyants et al. 2008), however the wider spread of the lateral inhibitory kernel may be the functional disynaptic expression of long distance horizontal excitatory connectivity impinging on local inhibitory neurons. Indeed, the excitatory neurons in the model also sent long-range connections. All connection probabilities were biased towards co-oriented neurons, but ignored the phase specificity of their targets, which implies that the model was not able to replicate the phase-specific relationship between excitatory and inhibitory conductances. Beyond this assertion it is difficult to assess how the model matched physiological measures at subthreshold levels as only the spiking output was analyzed and the model did not consider the spontaneous activity regime. Functionally, similarly to this study, the Somers et al. model was able to demonstrate the emergence of contrast-invariant orientation tuning, and contrast dependence of size tuning, but none of the other features of V1 processing considered here were examined.

Another similar pair of modeling studies was performed by Wielaard et al. (Wielaard and Sajda 2006a b) who also considered a large region of topologically organized cortex. These modeling studies, however, did not consider longrange cortical interactions, nor functional specificity of intra-cortical interactions, implying that the model was not able to replicate the phase-specific relationship between excitatory and inhibitory conductances. Wielaard et al. demonstrate the emergence of both Simple and Complex cell types and show how a bimodal distribution of modulation ratios at the level of spiking can emerge despite unimodally distributed modulation ratios of membrane potential. Furthermore, they show how such an only-locally-connected cortical model can explain the emergence of size tuning, quantitatively well matching experimental results. Interestingly, the authors do not demonstrate some of the more classical properties of V1 neurons, such as sharp mean orientation tuning (the limited data presented indicate a predominance of poorly tuned cells) or its contrast invariance. Similar to our model, Wielaard et al. demonstrate that the size tuning arises as a complex dynamic interplay between excitation and inhibition, whereby both conductances exhibit reduction at large stimulus sizes. Unfortunately Wielaard et al. do not assess the sub-threshold signals quantitatively, or characterize the background activity in the model. Finally, no ecological stimuli were tested in the model.

The Tononi group has also published a series of models focusing on the cat early visual system (Hill and Tononi 2005 Lumer et al. 1997b a Esser et al. 2007). While it isn't clear how much exactly the models differed across the studies, the manuscripts indicate that the models were similar and we will thus treat them as one for the purpose of 
bioRxiv preprint doi: https://doi.org/10.1101/416156; this version posted November 8, 2019. The copyright holder for this preprint (which was not certified by peer review) is the author/funder, who has granted bioRxiv a license to display the preprint in perpetuity. It is made available under aCC-BY 4.0 International license.

this discussion. In addition to the LGN and cortical layer 4 and 2/3 considered in the presented model, the Tononi et al. model series also incorporated a model of sub-granular layers and a rudimentary model of a generic extra-striate area that represented the totality of feedback input from higher-level visual areas to V1. While modeling neurons as point processes, Tononi et al. also considered a greater range of intrinsic conductance and synaptic types than the present study. The major difference between the studies is that of focus, where Tononi et al. aimed primarily at simulating the spontaneous activity during awake vs. slow wave sleep conditions. Accordingly, the model incorporated only rudimentary functionally specific circuitry, chiefly restricted to the classic Hubel \& Wiesel type convergence of thalamic afferents onto cells in granular and infragranular layer that induced the most basic form of orientation selectivity in the cortical neurons. Only two orthogonal orientations assigned to two spatially separated cell groups were considered. Beyond a single 3 second long presentation of a sine-grating stimulus that demonstrated differential activation of the two cell groups, no other investigation of evoked activity or any meaningful analysis of orientation selectivity was performed. On the other hand, the model demonstrated an impressive range of activity dynamics present in spontaneous awake and slow wave sleep brain states including spontaneous patterns of cortical activity, slow wave sleep mode with up \& down states, the slow oscillation propagation in cortical space, bi-modal distribution of $\mathrm{Vm}$ in sleep mode and gamma band synchronization in both sleep and awake modes.

Probably the most comprehensive V1 modeling effort prior to the present study is a series of modeling studies (Cai et al. 2005 Tao et al. 2006 Rangan et al. 2005) summarized in Rangan et al. (2009), architecturally very similar to the Wielaard et al. models $(2006,2006 a)$.While the models also focus on spontaneous regime dynamics, they rely on constant independent noisy spike input in the cortical neurons. In the multiple related models the authors have demonstrated a range of V1 properties in both spontaneous and evoked states including a fluctuation-driven spontaneous activity regime characterized by low firing rates (Cai et al. 2005), emergence of sharply tuned Simple and Complex cell types with a bimodally distributed modulation ratio of spike responses and unimodally distributed modulation ratio of membrane potential (Tao et al. 2006), and approximate contrast invariance of orientation tuning. The study also demonstrated two features of V1 computation not examined in our model: the spontaneous activity dynamics that are correlated with the intrinsic orientation organization of cortex (Cai et al. 2005), and the spatio-temporal patterns of cortical activity in response to a line-motion illusion stimulus (Rangan et al. 2005). Unlike in the present study, neither the contextual modulation nor naturalistic stimulation were explored in these models, nor was a detailed quantitative account of their sub-threshold behavior provided. Finally, while all the models summarized in Rangan et al. (2009) share a common architecture, it is not clear whether all these studies used identical parameterization of the models, and consequently if all these features could be achieved within a single model instance.

Finally, recently Chariker et al. (2016) have also made the case for a more comprehensive approach to the computational study of primary visual cortex, presenting a model that is, similarly to the present study, firmly grounded in anatomical data. The number of properties demonstrated in the model is, however, still limited, not exceeding the range presented in the series of studies of Wielaard et al. $(2006,2006 a)$ or those summarized in Rangan et al. (2009), although it is important to emphasize that, unlike those other model series, Chariker et al. (2016) explicitly demonstrate that all the claimed properties can emerge in a single model instance. The authors do not probe the model with any stimulus other than the ubiquitous drifting sinusoidal grating. The quantitative analysis of the model is limited: for 
example the orientation tuning in the model seems to be too broad, while characterization of the spontaneous state is missing. The model Simple cells also do not exhibit the anti-phase relationship between excitatory and inhibitory conductances. The authors do not investigate basic properties such as contrast invariance of orientation tuning, which, judging by the elevated firing of the example V1 cells in the cross-oriented condition, is missing. Finally, the analysis of model properties at the sub-threshold level is very limited.

Overall we believe the present study represents a significant advance towards providing a comprehensive integrative treatment of primary visual cortex. Together with the technological advances in building such complex integrative models in a modular manner, which we have fully shared with the community (Antolík and Davison 2013), and the principled approach to sharing the results and all relevant metadata from this study (http://v1model.arkheia.org), as well as the associated tools (Antolík and Davison 2018), we provide the most advanced platform for future long-term systematic incremental integrative research into primary visual cortex of higher mammals and beyond.

\section{Discussion}

Past models of $\mathrm{V} 1$ failed to exploit the full breadth of constraints imposed by experiments, and thus a coherent explanation for how V1 computations coexist within the underlying neural substrate is still lacking. We have approached this problem with a systematic, integrative, data-driven methodology, constructing a single model of $\mathrm{V} 1$ firmly grounded in anatomical and physiological data. We show that a single set of plausible connectivity principles-(i) weakly orientation-biased thalamic input, (ii) local, weakly push-pull-biased intra-Layer 4 connections, and (iii) long-range weakly orientation-specific intra-Layer $2 / 3$ connections-give rise to the most salient V1 computations.

The model provides insight into the underlying mechanisms of processing within CRF: orientation tuning of both spiking and subthreshold signals (Figures 6 and 7); emergence of Simple and Complex cells (Figure 8). The same model gives an accurate account of computations requiring integration beyond CRF: contrast dependent size tuning of both spikes and synaptic conductances (Figure 9). Finally, going beyond artificial stimuli, the model replicates the stimulus-dependent changes to precision of spiking responses, and to the trial-to-trial variability of Vm (Figure 10 ) when stimulated by a naturalistic movie. These stimulus evoked properties are underlaid by a resting state that is in very good quantitative agreement with physiological data (Figure 4).

\subsection{Novelty and predictions}

The central contribution of this study is the original convergence of the following key integrative principles: (i) the multitude of anatomical constraints respected, (ii) the multiplicity of neural signals (synaptic, single unit) and integration levels (conductance, neuronal, columns) validated, (iii) the multiplicity of the stimulation protocols tested, (iv) the diversity of functional phenomena explained, and ( $v$ ) the multiplicity of spatial and temporal scales validated. While many of the demonstrated properties have been modeled previously in isolation, we believe no previous model has been shown to reproduce such a broad range of V1 phenomena (see Table 2).

To the best of our knowledge, this model represents the first description of the asynchronous irregular resting 
regime that is quantitatively accurate at both supra- and sub-threshold level, self-consistent in the sense that it does not rely on any ad-hoc external noise input to cortex, and compatible with realistic evoked processing. This allows us to demonstrate, for the first time, how the trial-to-trial variability of $\mathrm{Vm}$ in $\mathrm{V} 1$ neurons can increase during drifting grating and decrease during naturalistic movie stimulation (Baudot et al. 2013).

This model also represents the first mechanistic explanation of orientation tuning that is fully consistent with the following physiological constraints: (i) orientation tuning is sharp across cortical layers (Cardin et al. 2007), (ii) inhibition is only moderately broader than excitation (Nowak et al. 2003 Cardin et al. 2007), and (iii) both DC and F1 components of spikes, $\mathrm{Vm}$ and conductances tend to be tuned to the cell's preferred orientation (Anderson et al. 2000) (although inhibitory conductance shows greater diversity of orientation tuning relative to spike-based preference (Fournier et al. 2014)). Many past models have only explored the orientation tuning of spiking responses (Hubel and \begin{tabular}{|l|l|l|l|l|l|l|}
\hline Wiesel 1962 & Pugh et al. 2000) and relied on either broad inhibition (Somers et al. 1995. Troyer et al. 1998 Chariker \\
\hline
\end{tabular} et al. 2016) or inhibition tuned to the orthogonal orientation (Sillito et al. 1980 Creutzfeldt et al. 1974), which is in contradiction with inhibitory conductances tuned to preferred orientation (Anderson et al. 2000). Few past models have been consistent with the constraint of sharply tuned responses of inhibitory cells and of inhibitory input tuned to the preferred orientation (Rubin et al. 2015). To the best of our knowledge no previous model has demonstrated the orientation tuning of both the DC and F1 components of all the sub-threshold signals.

The model also offers a number of testable predictions:

1. Layer- and cell-type-specific differences in resting regime:

(a) The spontaneous rates of inhibitory neurons are six times those of the excitatory ones.

(b) The spontaneous rates in Layer $2 / 3$ are lower than in Layer 4.

(c) There is greater synchrony among the inhibitory neurons.

2. Layer specific distributions of modulation ratios of spiking and $\mathrm{Vm}$ responses (layer specific data missing in cat).

3. Near contrast-invariance of inhibitory neurons.

4. Layer-specific differences in sub-threshold signal tuning.

5. Important testable assumptions were made about the model parameters:

(a) The sharpness of functional specificity of cortico-cortical connections in Layer 4.

(b) The excitatory-to-excitatory vs. excitatory-to-inhibitory pathway strength ratio.

\subsection{Other models of V1}

Models of orientation selectivity are typically categorized according to their circuitry ('feed-forward' vs 'recurrent'), the relative impact of such feed-forward vs recurrent contributions to the genesis of orientation selectivity, and the selectivity of the cortical inhibition. In 'feed-forward' models (Hubel and Wiesel 1962 Troyer et al. 1998 Ferster and Miller 2000) orientation tuning is induced by a strong sharply tuned thalamo-cortical pathway, while intra-cortical connections have only a minor influence. In contrast, 'recurrent' models (Ben-Yishai et al. 1995: Douglas et al. 1995) 
assume weak poorly tuned thalamic input but strong intra-cortical interactions. With respect to inhibition, the defining features are its orientation tuning, its presence or absence at orthogonal orientations, and its relationship to stimulus phase.

Although this opposition between thalamic-driven and cortical-recurrent computing schemes has long dominated the literature, this dichotomic categorization is highly oversimplified (Teich and Qian 2006). Following the anatomical and physiological evidence to determine the model architecture, as we have done here, results in a model that resides in between these standard classes. The thalamo-cortical connections in the model constitute a fraction of the synaptic input to Layer $4(\sim 10 \%)$, which would assign the model to the recurrent category. But the dominating intra-cortical connections in Layer 4 are biased towards a push-pull organization, typically assumed in feed-forward models (Troyer et al. 1998). Although, based on experimental evidence (Buzás et al. 2006 Ko et al. 2011), this functional bias is weaker than in previous models. Consequently, the inhibitory input in layer 4 neurons is broad and present at orthogonal orientations. But the resulting orientation tuning of inhibitory neurons is sharp, comparable to that of excitatory ones, showing that even weak and poorly orientation-tuned afferent input and broad inhibitory input can lead to sharply tuned inhibitory neurons, in line with experiments (Cardin et al. 2007 Anderson et al. 2000). Furthermore, in classical feed-forward models the DC component of the feed-forward excitatory input is canceled-out by the DC component of the inhibition (Troyer et al. 1998). It is the F1 component of the $\mathrm{Vm}$ that induces the orientation tuning. But, in line with experimental evidence, our model exhibits orientation tuning of the DC components of both excitatory and inhibitory conductances and also of the $\mathrm{Vm}$, suggestive of boosting of mean response at preferred orientations typical of recurrent models. However, the push-pull interactions remain an important factor in generation of contrast-invariant orientation tuning, as evidenced by the dominance of the F1 components in Simple cells. This shows that the different strategies of generating orientation tuning can co-exist within the same neural circuit, while being consistent with a broader range of physiological findings, exemplifying the benefits of the data-driven integrative approach to counteract fragmentation of knowledge.

Recently, a feed-forward explanation of contrast-invariant orientation tuning was proposed by Finn et al. (2007) relying on two key mechanisms: an expansive non-linearity governing the relationship between $\mathrm{Vm}$ and the spike rate, and the contrast dependence of the thalamic input. However, although the formulation of the Finn et al. model is feedforward, the assumed non-linearities may in the cortical substrate be implemented by recurrent dynamics. Furthermore, the Finn et al. model does not explain the fact that the modulation of the $\mathrm{Vm}$ is dominated by inhibition, and the anti-phase relationship between inhibition and excitation (Hirsch et al. 1998, Anderson et al. 2000 Monier et al. 2008).

Finally, a recent study from the Allen Institute (Arkhipov et al. 2018) also followed a comprehensive, data-driven approach, employed a broad set of test protocols, and demonstrated orientation selectivity, amplification of thalamic inputs, presence of gamma oscillations, and log-normal distributions of firing rates. Unlike the present study it did not investigate more complex functional properties, such as size-tuning or trial-to-trial precision. The Arkhipov et al. model is complementary to the model presented here: cat V1 in the anesthetized state vs awake mice (Arkhipov et al. 2018) (respective merits of the two preparations are discussed in section 2.1). The Arkhipov et al. study incorporated also neural morphology. Interestingly, they showed that a simplified, integrate-and-fire version of the model remained in a very good agreement with in-vivo data, justifying the level of detail chosen here. 


\subsection{Future work}

This model is meant as the first step in a long-term systematic effort to build a single comprehensive model of V1 and beyond. We chose V1 as the first target because it is the most thoroughly studied cortical area, with a breadth of experimental findings ripe for knowledge consolidation. In section 2.8 we listed the most important features that were omitted due to the need to proceed in manageable steps of complexity, but all these omissions are natural candidates for future work. To that end, work is in progress in our group to integrate cortico-thalamic feedback (Guarino et al. 2017).

\section{References}

Abbott LF. 1997. Synaptic Depression and Cortical Gain Control. Science 275:221-224.

Akasaki T, Sato H, Yoshimura Y, Ozeki H and Shimegi S. 2002. Suppressive effects of receptive field surround on neuronal activity in the cat primary visual cortex. Neurosci Res 43:207-20.

Alitto HJ and Usrey WM. 2004. Influence of contrast on orientation and temporal frequency tuning in ferret primary visual cortex. Journal of Neurophysiology 91:2797-2808.

Allen C and Stevens CF. 1994. An evaluation of causes for unreliability of synaptic transmission. Proceedings of the National Academy of Sciences of the United States of America 91:10380-10383.

Allen EA and Freeman RD. 2006. Dynamic spatial processing originates in early visual pathways. Journal of Neuroscience $26: 11763-11774$.

Allen Brain Observatory contributors . 2016. Allen Institute for Brain Science: Allen Brain Observatory. http://observatory.brain-map.org/visualcoding. [Online; accessed 17-July-2018].

Anderson JS, Carandini M and Ferster D. 2000. Orientation tuning of input conductance, excitation, and inhibition in cat primary visual cortex. Journal of Neurophysiology 84:909-926.

Anderson JS, Lampl I, Gillespie DC and Ferster D. 2001. Membrane potential and conductance changes underlying length tuning of cells in cat primary visual cortex. Journal of Neuroscience 21:2104-2112.

Angelucci A and Bullier J. 2003. Reaching beyond the classical receptive field of V1 neurons: Horizontal or feedback axons? Journal of Physiology Paris 97:141-154.

Angelucci A, Levitt JB, Walton EJ, Hupé JM, Bullier J and Lund JS. 2002. Circuits for local and global signal integration in primary visual cortex. Journal of Neuroscience 22:8633-8646.

Antolík J and Bednar JAJ. 2011. Development of Maps of Simple and Complex Cells in the Primary Visual Cortex. Frontiers in Computational Neuroscience 5:1-19. 
Antolík J and Davison AA. 2018. Arkheia: Data Management and Communication for Open Computational Neuroscience. Frontiers in Neuroinformatics 12:6.

Antolík J and Davison AP. 2013. Integrated workflows for spiking neuronal network simulations. Frontiers in Neuroinformatics 7:1-15.

Arkhipov A, Gouwens NW, Billeh YN, Gratiy S, lyer R, Wei Z, Xu Z, Abbasi-Asl R, Berg J, Buice M, Cain N, da Costa N, de Vries S, Denman D, Durand S, Feng D, Jarsky T, Lecoq J, Lee B, Li L, Mihalas S, Ocker GK, Olsen SR, Reid RC, Soler-Llavina G, Sorensen SA, Wang Q, Waters J, Scanziani M and Koch C. 2018. Visual physiology of the layer 4 cortical circuit in silico. PLOS Computational Biology 14:e1006535.

Ascoli GA, Donohue DE and Halavi M. 2007. NeuroMorpho.Org: A Central Resource for Neuronal Morphologies. Journal of Neuroscience 27:9247-9251.

Banitt Y, Martin KA and Segev I. 2007. A biologically realistic model of contrast invariant orientation tuning by thalamocortical synaptic depression. Journal of Neuroscience 27:10230-10239.

Bardy C, Huang JY, Wang C, FitzGibbon T and Dreher B. 2006. 'Simplification' of responses of complex cells in cat striate cortex: Suppressive surrounds and 'feedback' inactivation. Journal of Physiology 574:731-750.

Baudot P, Levy M, Marre O, Monier C, Pananceau M and Frégnac Y. 2013. Animation of natural scene by virtual eye-movements evokes high precision and low noise in V1 neurons. Frontiers in Neural Circuits 7:206.

Beaulieu C and Colonnier M. 1985. A laminar analysis of the number of round asymmetrical and flat symmetrical synapses on spines, dendritic trunks, and cell bodies in area 17 of the cat. Journal of Comparative Neurology 231:180-189.

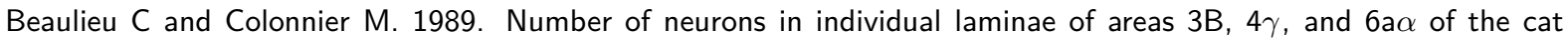
cerebral cortex: A comparison with major visual areas. Journal of Comparative Neurology 279:228-234.

Beaulieu C, Kisvarday Z, Somogyi P, Cynader M and Cowey A. 1992. Quantitative distribution of gaba-immunopositive and -immunonegative neurons and synapses in the monkey striate cortex (area 17). Cerebral Cortex 2:295-309.

Beltramo R and Scanziani M. 2019. A collicular visual cortex: Neocortical space for an ancient midbrain visual structure. Science 363:64-69.

Ben-Yishai R, Lev Bar-Or R and Sompolinsky H. 1995. Theory of orientation tuning in visual cortex. Proceedings of the National Academy of Sciences of the United States of America 92:3844-3848.

Binzegger T, Douglas RJ and Martin KA. 2004. A quantitative map of the circuit of cat primary visual cortex. Journal of Neuroscience 24:8441-8453.

Bonin V, Mante V and Carandini M. 2005. The suppressive field of neurons in lateral geniculate nucleus. Journal of Neuroscience 25:10844-10856. 
Bringuier V, Chavane F, Glaeser L and Frégnac Y. 1999. Horizontal propagation of visual activity in the synaptic integration field of area 17 neurons. Science 283:695-699.

Bringuier V, Frégnac Y, Baranyi A, Debanne D and Shulz DE. 1997. Synaptic origin and stimulus dependency of neuronal oscillatory activity in the primary visual cortex of the cat. Journal of Physiology 500:751-774.

Brunel N. 2000. Dynamics of networks of randomly connected excitatory and inhibitory spiking neurons. Journal of Physiology-Paris 94:445-463.

Budd JM. 1998. Extrastriate feedback to primary visual cortex in primates: A quantitative analysis of connectivity. Proceedings of the Royal Society B: Biological Sciences 265:1037-1044.

Budd JM and Kisvárday ZF. 2001. Local lateral connectivity of inhibitory clutch cells in layer 4 of cat visual cortex (area 17). Experimental Brain Research 140:245-250.

Buzás P, Kovács K, Ferecskó AS, Budd JM, Eysel UT and Kisvárday ZF. 2006. Model-based analysis of excitatory lateral connections in the visual cortex. Journal of Comparative Neurology 499:861-881.

Buzsáki G and Mizuseki K. 2014. The log-dynamic brain: how skewed distributions affect network operations. Nature Reviews Neuroscience 15:264-278.

Cai D, Rangan AV and McLaughlin DW. 2005. Architectural and synaptic mechanisms underlying coherent spontaneous activity in V1. Proceedings of the National Academy of Sciences of the United States of America 102:5868-5873.

Cardin JA, Palmer LA and Contreras D. 2007. Stimulus feature selectivity in excitatory and inhibitory neurons in primary visual cortex. Journal of Neuroscience 27:10333-10344.

Cavaggioni A. 1968. The dark-discharge of the eye in the unrestrained cat. Pflügers Archiv European Journal of Physiology 304:75-80.

Cavanaugh JR, Bair W and Anthony Movshon J. 2002. Nature and interaction of signals from the receptive field center and surround in macaque V1 neurons. Journal of Neurophysiology 88:2530-2546.

Chariker L, Shapley R and Young LS. 2016. Orientation selectivity from very sparse LGN inputs in a comprehensive model of macaque V1 cortex. Journal of Neuroscience 36:12368-12384.

Chavane F, Sharon D, Jancke D, Marre O, Frégnac Y and Grinvald A. 2011. Lateral spread of orientation selectivity in V1 is controlled by intracortical cooperativity. Frontiers in Systems Neuroscience 5:1-26.

Cossart R, Aronov D and Yuste R. 2003. Attractor dynamics of network UP states in the neocortex. Nature 423:283288.

Creutzfeldt OD, Kuhnt $U$ and Benevento LA. 1974. An intracellular analysis of visual cortical neurones to moving stimuli: Responses in a co-operative neuronal network. Experimental Brain Research 21:251-274. 
Croner LJ, Purpura K and Kaplan E. 1993. Response variability in retinal ganglion cells of primates. Proceedings of the National Academy of Sciences of the United States of America 90:8128-8130.

Cruikshank SJ, Lewis TJ and Connors BW. 2007. Synaptic basis for intense thalamocortical activation of feedforward inhibitory cells in neocortex. Nature Neuroscience 10:462-468.

Da Costa NM and Martin KA. 2011. How thalamus connects to spiny stellate cells in the cat's visual cortex. Journal of Neuroscience 31:2925-2937.

Destexhe A. 2009. Self-sustained asynchronous irregular states and Up-Down states in thalamic, cortical and thalamocortical networks of nonlinear integrate-and-fire neurons. Journal of Computational Neuroscience 27:493-506.

Destexhe A and Rudolph-Lilith M. 2012. Neuronal noise. Springer Series in Computational Neuroscience. Springer US.

Douglas R, Koch C, Mahowald M, Martin K and Suarez H. 1995. Recurrent excitation in neocortical circuits. Science 269:981-985.

Einevoll GT, Destexhe A, Diesmann M, Grün S, Jirsa V, de Kamps M, Migliore M, Ness TV, Plesser HE and Schürmann F. 2019. The Scientific Case for Brain Simulations.

Esposito F, Mulert C and Goebel R. 2009. Combined distributed source and single-trial EEG-fMRI modeling: Application to effortful decision making processes. Neurolmage 47:112-121.

Esser SK, Hill SL and Tononi G. 2007. Sleep homeostasis and cortical synchronization: I. Modeling the effects of synaptic strength on sleep slow waves. Sleep 30:1617-1630.

Ferster D and Miller KD. 2000. Neural Mechanisms of Orientation Selectivity in the Visual Cortex. Annual Review of Neuroscience 23:441-471.

Field GD and Rieke F. 2002. Mechanisms regulating variability of the single photon responses of mammalian rod photoreceptors. Neuron 35:733-747.

Finn IM, Priebe NJ and Ferster D. 2007. The emergence of contrast-invariant orientation tuning in simple cells of cat visual cortex. Neuron 54:137-52.

Fourcaud-Trocmé N, Hansel D, van Vreeswijk C and Brunel N. 2003. How spike generation mechanisms determine the neuronal response to fluctuating inputs. Journal of Neuroscience 23:11628-40.

Fournier J, Monier C, Levy M, Marre O, Sari K, Kisvarday ZF and Fregnac Y. 2014. Hidden Complexity of Synaptic Receptive Fields in Cat V1. Journal of Neuroscience 34:5515-5528.

Frégnac Y. 2012. Reading Out the Synaptic Echoes of Low-Level Perception in V1, pp. 486-495. In A. Fusiello, V. Murino, and R. Cucchiara (eds.), Lecture Notes in Computer Science 1, volume 7583 of Lecture Notes in Computer Science. Springer. 
Frégnac Y. 2017. Big data and the industrialization of neuroscience: A safe roadmap for understanding the brain? Science 358:470-477.

Frégnac Y and Bathellier B. 2015. Cortical Correlates of Low-Level Perception: From Neural Circuits to Percepts. Neuron 88:110-126.

Gerard-Mercier F, Carelli PV, Pananceau M, Troncoso XG and Frégnac Y. 2016. Synaptic correlates of low-level perception in V1. Journal of Neuroscience 36:3925-3942.

Gewaltig MO and Diesmann M. 2007. NEST (NEural Simulation Tool). Scholarpedia 2:1430.

Ghose GM and Freeman RD. 1992. Oscillatory discharge in the visual system: Does it have a functional role? Journal of Neurophysiology 68:1558-1574.

Guarino D, Antolík J, Davison AP and Frégnac Y. 2017. An integrative model explaining many functions of corticothalamic feedback. In 26th Annual Computational Neuroscience Meeting (CNS* 2017): Part 2, volume 18, p. 59. BMC Neuroscience.

Harris KD and Mrsic-Flogel TD. 2013. Cortical connectivity and sensory coding.

Hill S and Tononi G. 2005. Modeling sleep and wakefulness in the thalamocortical system. Journal of neurophysiology 93:1671-98.

Hirsch Ja, Alonso JM, Reid RC and Martinez LM. 1998. Synaptic Integration in Striate Cortical Simple Cells. Journal of Neuroscience 18:9517-9528.

Hirsch JA and Martinez LM. 2006. Laminar processing in the visual cortical column. Current Opinion in Neurobiology 16:377-384.

Hoffmann JH, Meyer HS, Schmitt AC, Straehle J, Weitbrecht T, Sakmann B and Helmstaedter M. 2015. Synaptic conductance estimates of the connection between local inhibitor interneurons and pyramidal neurons in layer $2 / 3$ of a cortical column. Cerebral Cortex 25:4415-4429.

Hromadka T, DeWeese MR and Zador AM. 2008. Sparse Representation of Sounds in the Unanesthetized Auditory Cortex. PLoS biology 6:124-137.

Hubel DH and Wiesel TN. 1962. Receptive fields, binocular interaction and functional architecture in the cat's visual cortex. The Journal of Physiology 160:106-154.

Ichida JM, Schwabe L, Bressloff PC and Angelucci A. 2007. Response Facilitation From the "Suppressive" Receptive Field Surround of Macaque V1 Neurons. Journal of Neurophysiology 98:2168-2181.

Johnson RR and Burkhalter A. 1996. Microcircuitry of forward and feedback connections within rat visual cortex. Journal of Comparative Neurology 368:383-398. 
Jones HE, Andolina IM, Oakely NM, Murphy PC and Sillito AM. 2000. Spatial summation in lateral geniculate nucleus and visual cortex. Experimental Brain Research 135:279-284.

Jones HE, Grieve KL, Wang W and Sillito aM. 2001. Surround suppression in primate V1. Journal of Neurophysiology 86:2011-2028.

Jones JP and Palmer LA. 1987. The two-dimensional spatial structure of simple receptive fields in cat striate cortex. Journal of Neurophysiology 58:1187-1211.

Kayser AS and Miller KD. 2002. Opponent Inhibition: A Developmental Model of Layer 4 of the Neocortical Circuit. Neuron 33:131-142.

Ko H, Hofer SB, Pichler B, Buchanan KA, Sjöström PJ and Mrsic-Flogel TD. 2011. Functional specificity of local synaptic connections in neocortical networks. Nature 473:87-91.

Kremkow J, Jin J, Komban SJ, Wang Y, Lashgari R, Li X, Jansen M, Zaidi Q and Alonso JMJM. 2014. Neuronal nonlinearity explains greater visual spatial resolution for darks than lights. Proceedings of the National Academy of Sciences of the United States of America 111:3170-5.

Kremkow J, Perrinet LU, Monier C, Alonso JM, Aertsen A, Frégnac Y and Masson GS. 2016. Push-pull receptive field organization and synaptic depression: Mechanisms for reliably encoding naturalistic stimuli in V1. Frontiers in Neural Circuits 10.

Kumar A, Schrader S, Aertsen A and Rotter S. 2008. The high-conductance state of cortical networks. Neural Computation 20:1-43.

Lauritzen TZ and Miller KD. 2003. Different roles for simple-cell and complex-cell inhibition in V1. Journal of Neuroscience 23:10201-10213.

Le Van Quyen M, Muller LE, Telenczuk B, Halgren E, Cash S, Hatsopoulos NG, Dehghani N and Destexhe A. 2016. High-frequency oscillations in human and monkey neocortex during the wake-sleep cycle. Proceedings of the National Academy of Sciences 113:9363-9368.

Lee AK, Manns ID, Sakmann B and Brecht M. 2006. Whole-Cell Recordings in Freely Moving Rats. Neuron 51:399-407.

Lee WCA, Bonin V, Reed M, Graham BJ, Hood G, Glattfelder K and Reid RC. 2016. Anatomy and function of an excitatory network in the visual cortex. Nature 532:370-374.

Levine MW. 1991. The distribution of the intervals between neural impulses in the maintained discharges of retinal ganglion cells. Biological Cybernetics 65:459-467.

Lumer ED, Edelman GM and Tononi G. 1997a. Neural dynamics in a model of the thalamocortical system . II. The role of neural synchrony tested through perturbations of spike timing. Cerebral Cortex 7:228-236. 
Lumer ED, Edelman GM and Tononi G. 1997b. Neural dynamics in a model of the thalamocortical system. I. Layers, loops and the emergence of fast synchronous rhythms. Cerebral Cortex 7:207-27.

Markov NT, Vezoli J, Chameau P, Falchier A, Quilodran R, Huissoud C, Lamy C, Misery P, Giroud P, Ullman S, Barone P, Dehay C, Knoblauch K and Kennedy H. 2014. Anatomy of hierarchy: Feedforward and feedback pathways in macaque visual cortex. Journal of Comparative Neurology 522:225-259.

Markram H, Muller E, Ramaswamy S, Reimann MW, Abdellah M, Sanchez CA, Ailamaki A, Alonso-Nanclares L, Antille N, Arsever S, Kahou GAA, Berger TK, Bilgili A, Buncic N, Chalimourda A, Chindemi G, Courcol JD, Delalondre F, Delattre V, Druckmann S, Dumusc R, Dynes J, Eilemann S, Gal E, Gevaert ME, Ghobril JP, Gidon A, Graham JW, Gupta A, Haenel V, Hay E, Heinis T, Hernando JB, Hines M, Kanari L, Keller D, Kenyon J, Khazen G, Kim Y, King JG, Kisvarday Z, Kumbhar P, Lasserre S, Le Bé JV, Magalhães BRC, Merchán-Pérez A, Meystre J, Morrice BR, Muller J, Muñoz-Céspedes A, Muralidhar S, Muthurasa K, Nachbaur D, Newton TH, Nolte M, Ovcharenko A, Palacios J, Pastor L, Perin R, Ranjan R, Riachi I, Rodríguez JR, Riquelme JL, Rössert C, Sfyrakis K, Shi Y, Shillcock JC, Silberberg G, Silva R, Tauheed F, Telefont M, Toledo-Rodriguez M, Tränkler T, Van Geit W, Díaz JV, Walker R, Wang Y, Zaninetta SM, Defelipe J, Hill SL, Segev I and Schürmann F. 2015. Reconstruction and Simulation of Neocortical Microcircuitry. Cell 163:456-492.

Markram H, Toledo-Rodriguez M, Wang Y, Gupta A, Silberberg G and Wu C. 2004. Interneurons of the neocortical inhibitory system. Nature Reviews Neuroscience 5:793-807.

Markram H, Wang Y and Tsodyks M. 1998. Differential signaling via the same axon of neocortical pyramidal neurons. Proceedings of the National Academy of Sciences of the United States of America 95:5323-5328.

Martinez LM, Wang Q, Reid RC, Pillai C, Alonso JM, Sommer FT and Hirsch JA. 2005. Receptive field structure varies with layer in the primary visual cortex. Nature Neuroscience 8:372-379.

McCormick DA, Connors BW, Lighthall JW and Prince DA. 1985. Comparative electrophysiology of pyramidal and sparsely spiny stellate neurons of the neocortex. Journal of Neurophysiology 54:782-806.

Monier C, Chavane F, Baudot P, Graham LJ and Frégnac Y. 2003. Orientation and direction selectivity of synaptic inputs in visual cortical neurons: a diversity of combinations produces spike tuning. Neuron 37:663-80.

Monier C, Fournier J and Frégnac Y. 2008. In vitro and in vivo measures of evoked excitatory and inhibitory conductance dynamics in sensory cortices. Journal of Neuroscience Methods 169:323-365.

Movshon JA, Thompson ID and Tolhurst DJ. 1978. Spatial and temporal contrast sensitivity of neurones in areas 17 and 18 of the cat's visual cortex. The Journal of Physiology 283:101-120.

Naud R, Marcille N, Clopath C and Gerstner W. 2008. Firing patterns in the adaptive exponential integrate-and-fire model. Biological Cybernetics 99:335-347.

Nowak LG, Azouz R, Sanchez-Vives MV, Gray CM and McCormick Da. 2003. Electrophysiological classes of cat primary visual cortical neurons in vivo as revealed by quantitative analyses. Journal of Neurophysiology 89:1541-1566. 
Nowak LG, Sanchez-Vives MV and McCormick DA. 2008. Lack of orientation and direction selectivity in a subgroup of fast-spiking inhibitory interneurons: Cellular and synaptic mechanisms and comparison with other electrophysiological cell types. Cerebral Cortex 18:1058-1078.

Ohana O, Portner H and Martin KAC. 2012. Fast Recruitment of Recurrent Inhibition in the Cat Visual Cortex. PLoS ONE 7:e40601.

Okamoto M, Naito T, Sadakane O, Osaki H and Sato H. 2009. Surround suppression sharpens orientation tuning in the cat primary visual cortex. European Journal of Neuroscience 29:1035-1046.

Olshausen BA. 2013. 20 years of learning about vision: Questions answered, questions unanswered, and questions not yet asked, pp. 243-270. In 20 Years of Computational Neuroscience. Springer.

Olshausen BA and Field DJ. 1996. Emergence of simple-cell receptive field properties by learning a sparse code for natural images. Nature 381:607-609.

Ozeki H, Finn IM, Schaffer ES, Miller KD and Ferster D. 2009. Inhibitory Stabilization of the Cortical Network Underlies Visual Surround Suppression. Neuron 62:578-592.

Papaioannou J and White A. 1972. Maintained activity of lateral geniculate nucleus neurons as a function of background luminance. Experimental Neurology 34:558-566.

Payne B and Peters A. 2001. The Cat Primary Visual Cortex. Elsevier Science.

Pfeffer CK, Xue M, He M, Huang ZJ and Scanziani M. 2013. Inhibition of inhibition in visual cortex: The logic of connections between molecularly distinct interneurons. Nature Neuroscience 16:1068-1076.

Potjans TC and Diesmann M. 2014. The cell-type specific cortical microcircuit: Relating structure and activity in a full-scale spiking network model. Cerebral Cortex 24:785-806.

Priebe NJ, Mechler F, Carandini M and Ferster D. 2004. The contribution of spike threshold to the dichotomy of cortical simple and complex cells. Nature Neuroscience 7:1113-1122.

Pugh M, Ringach D, Shapley R and Shelley M. 2000. Computational Modeling of Orientation Tuning Dynamics in V1 Neurons. J. Comp. Neuroscience 8:143-159.

Rangan A, Tao L, Kovacic G and Cai D. 2009. Multiscale modeling of the primary visual cortex. IEEE Engineering in Medicine and Biology Magazine 28:19-24.

Rangan AV, Cai D and McLaughlin DW. 2005. Modeling the spatiotemporal cortical activity associated with the line-motion illusion in primary visual cortex. Proceedings of the National Academy of Sciences of the United States of America 102:18793-18800.

Ringach DL. 2004. Mapping receptive fields in primary visual cortex. Journal of Physiology 558:717-728. 
Rogala J, Waleszczyk WJ, Wróbel A and Wójcik DK. 2013. Effect of cortex inactivation on spontaneous activity of cells in perigeniculate and dorsal lateral geniculate nuclei. BMC Neuroscience 14:P418.

Rubin DB, VanHooser SD and Miller KD. 2015. The stabilized supralinear network: A unifying circuit motif underlying multi-input integration in sensory cortex. Neuron 85:402-417.

Rust NC and Movshon JA. 2005. In praise of artifice. Nature Neuroscience 8:1647-1650.

Salari V, Scholkmann F, Bokkon I, Shahbazi F and Tuszynski J. 2016. The Physical Mechanism for Retinal Discrete Dark Noise: Thermal Activation or Cellular Ultraweak Photon Emission? PLoS ONE 11:1-16.

Sceniak MP, Ringach DL, Hawken MJ and Shapley R. 1999. Contrast's effect on spatial summation by macaque V1 neurons. Nature Neuroscience 2:733-739.

Sejnowski TJ, Koch C and Churchland PS. 1988. Computational Neuroscience. Science 241:1299-1306.

Sengpiel F, Sen A and Blakemore C. 1997. Characteristics of surround inhibition in cat area 17. Experimental Brain Research 116:216-228.

Shu Y, Hasenstaub A and McCormick DA. 2003. Turning on and off recurrent balanced cortical activity. Nature 423:288-293.

Sillito AM, Jones HE, Gerstein GL and West DC. 1994. Feature-linked synchronization of thalamic relay cell firing induced by feedback from the visual cortex. Nature 369:479-482.

Sillito AM, Kemp JA, Milson JA and Berardi N. 1980. A re-evaluation of the mechanisms underlying simple cell orientation selectivity. Brain Research 194:517-520.

Skottun BC, De Valois RL, Grosof DH, Movshon JA, Albrecht DG and Bonds AB. 1991. Classifying simple and complex cells on the basis of response modulation. Vision Research 31:1078-1086.

Somers DC, Nelson SB and Sur M. 1995. An emergent model of orientation selectivity in cat visual cortical simple cells. Journal of Neuroscience 15:5448-5465.

Somers DC, Todorov EV, Siapas AG, Toth LJ, Kim DS and Sur M. 1998. A local circuit approach to understanding integration of long-range inputs in primary visual cortex. Cerebral Cortex 8:204-217.

Stepanyants A, Hirsch JA, Martinez LM, Kisvárday ZF, Ferecskó AS and Chklovskii DB. 2008. Local potential connectivity in cat primary visual cortex. Cerebral Cortex 18:13-28.

Stepanyants A, Martinez LM, Ferecsko AS and Kisvarday ZF. 2009. The fractions of short- and long-range connections in the visual cortex. Proceedings of the National Academy of Sciences 106:3555-3560.

Stratford KJ, Tarczy-Hornoch K, Martin KA, Bannister NJ and Jack JJ. 1996. Excitatory synaptic inputs to spiny stellate cells in cat visual cortex. Nature 382:258-261. 
Swadlow HA. 2003. Fast-spike Interneurons and Feedforward Inhibition in Awake Sensory Neocortex. Cerebral Cortex 13:25-32.

Tailby C, Solomon SG, Peirce JW and Metha AB. 2007. Two expressions of "surround suppression" in V1 that arise independent of cortical mechanisms of suppression. Visual Neuroscience 24:99-109.

Tao L, Cai D, McLaughlin DW, Shelley MJ and Shapley R. 2006. Orientation selectivity in visual cortex by fluctuationcontrolled criticality. Proceedings of the National Academy of Sciences of the United States of America 103:1291112916.

Teich AF and Qian N. 2006. Comparison among some models of orientation selectivity. Journal of Neurophysiology 96:404-419.

Thomson AM. 2003. Interlaminar Connections in the Neocortex. Cerebral Cortex 13:5-14.

Thomson AM. 2010. Neocortical layer 6, a review. Frontiers in neuroanatomy 4:13.

Troyer TW, Krukowski aE, Priebe NJ and Miller KD. 1998. Contrast-invariant orientation tuning in cat visual cortex: thalamocortical input tuning and correlation-based intracortical connectivity. The Journal of Neuroscience 18:59085927.

Tusa RJ, Palmer La and Rosenquist aC. 1978. The retinotopic organization of area 17 (striate cortex) in the cat. The Journal of comparative neurology 177:213-35.

Van Vreeswijk C and Sompolinsky H. 1996. Chaos in neuronal networks with balanced excitatory and inhibitory activity. Science 274:1724-1726.

Vogels TP and Abbott LF. 2005. Signal propagation and logic gating in networks of integrate-and-fire neurons. Journal of Neuroscience 25:10786-10795.

Wallace DJ, Greenberg DS, Sawinski J, Rulla S, Notaro G and Kerr JN. 2013. Rats maintain an overhead binocular field at the expense of constant fusion. Nature 498:65-69.

Wang C, Bardy C, Huang JY, FitzGibbon T and Dreher B. 2009. Constrast dependence of center and surround integration in primary visual cortex of the cat. Journal of Vision 9:20.1-15.

Wang W, Jones HE, Andolina IM, Salt TE and Sillito AM. 2006. Functional alignment of feedback effects from visual cortex to thalamus. Nature Neuroscience 9:1330-1336.

Wang Y, Jin J, Kremkow J, Lashgari R, Komban SJ and Alonso JM. 2014. Columnar organization of spatial phase in visual cortex. Nature Neuroscience 18:97-103.

Wielaard J and Sajda P. 2006a. Circuitry and the classification of simple and complex cells in V1. Journal of Neurophysiology 96:2739-2749. 
bioRxiv preprint doi: https://doi.org/101101/416156; this version posted November 8, 2019. The copyright holder for this preprint (which

was not certified by peer review) is the author/funder, who has granted bioRxiv a license to display the preprint in perpetuity. It is made available under aCC-BY 4.0 International license.

Wielaard J and Sajda P. 2006b. Extraclassical receptive field phenomena and short-range connectivity in V1. Cerebral Cortex 16:1531-1545.

Wilson JR and Sherman SM. 1976. Receptive-field characteristics of neurons in cat striate cortex: Changes with visual field eccentricity. Journal of Neurophysiology 39:512-533.

Xu X and Callaway EM. 2009. Laminar specificity of functional input to distinct types of inhibitory cortical neurons. Journal of Neuroscience 29:70-85.

Zhu M and Rozell CJ. 2013. Visual Nonclassical Receptive Field Effects Emerge from Sparse Coding in a Dynamical System. PLoS Computational Biology 9:1-15.

Ziskind A. 2013. Neurons in Cat Primary Visual Cortex cluster by degree of tuning but not by absolute spatial phase or temporal response phase. $\mathrm{PhD}$ Thesis . 